\title{
3D PRINTED BIOCERAMICS FOR DUAL ANTIBIOTIC DELIVERY TO TREAT IMPLANT-ASSOCIATED BONE INFECTION
}

\author{
J.A. Inzana ${ }^{1,2}$, R.P. Trombetta ${ }^{1,2}$, E.M. Schwarz ${ }^{1,2,3}$, S.L. Kates ${ }^{1,3}$ and H.A. Awad ${ }^{1,2,3, *}$ \\ ${ }^{1}$ Center for Musculoskeletal Research, University of Rochester Medical Center, 601 Elmwood Avenue, Box 665. \\ Rochester, NY 14642, USA \\ ${ }^{2}$ Department of Biomedical Engineering, University of Rochester, 207 Robert B. Goergen Hall, Rochester, \\ NY 14642, USA \\ ${ }^{3}$ Department of Orthopedics, University of Rochester Medical Center, 601 Elmwood Avenue, Rochester, \\ NY 14642, USA
}

\begin{abstract}
Surgical implant-associated bone infections (osteomyelitis) have severe clinical and socioeconomic consequences. Treatment of chronic bone infections often involves antibiotics given systemically and locally to the affected site in poly (methyl methacrylate) (PMMA) bone cement. Given the high antibiotic concentrations required to affect bacteria in biofilm, local delivery is important to achieve high doses at the infection site. PMMA is not suitable to locally-deliver some biofilm-specific antibiotics, including rifampin, due to interference with PMMA polymerisation. To examine the efficacy of localised, combinational antibiotic delivery compared to PMMA standards, we fabricated rifampin- and vancomycin-laden calcium phosphate scaffolds (CPS) by three-dimensional (3D) printing to treat an implant-associated Staphylococcus aureus bone infection in a murine model.

All vancomycin- and rifampin-laden CPS treatments significantly reduced the bacterial burden compared with vancomycin-laden PMMA. The bones were bacteria culture negative in $50 \%$ of the mice that received sustained release vancomycin- and rifampin-laden CPS. In contrast, $100 \%$ of the bones treated with vancomycin monotherapy using PMMA or CPS were culture positive. Yet, the monotherapy CPS significantly reduced the bacterial metabolic load following revision compared to PMMA. Biofilm persisted on the fixation hardware, but the infection-induced bone destruction was significantly reduced by local rifampin delivery.

These data demonstrate that, despite the challenging implant-retaining infection model, co-delivery of rifampin and vancomycin from 3D printed CPS, which is not possible with PMMA, significantly improved the outcomes of implant-associated osteomyelitis. However, biofilm persistence on the fixation hardware reaffirms the importance of implant exchange or other biofilm eradication strategies to complement local antibiotics.
\end{abstract}

Keywords: Bone, Staphylococcus aureus, osteomyelitis, three dimensional printing, calcium phosphate, ceramics, antibiotics, drug delivery, Vancomycin, Rifampin.

*Address for correspondence:

Hani A. Awad, Ph.D.

University of Rochester Medical Center

601 Elmwood Avenue, Box 665

Rochester, NY 14642, USA

Telephone number: $+1-585-273-5268$

Fax number: +1-585-276-2177

Email: hani_awad@urmc.rochester.edu
The biofilm-forming species Staphylococcus aureus ( $S$. aureus) is the most common pathogen in implant-associated infections. These infections are extremely difficult to treat due to the ability of the pathogens in biofilm to resist the immune response and antibiotic treatments, leading to the highest re-infection rates (Darouiche, 2004; Keren et al., 2004; Rani et al., 2007; Yun et al., 2008; Zimmerli et al., 2004). Most often, these infections are associated with bone fracture fixation devices, such as plates and screws, which account for over two-thirds of all surgical implant infections with an estimated 100,000 cases per year in the USA alone, and a cost of $\$ 15,000-50,000$ per incident (Darouiche, 2004). When revision surgery is required for infection management, surgeons sometimes elect to retain the orthopaedic implant for an acute ( $<3$ weeks) infection case if it is biomechanically stable within the bone, the soft tissue is in good condition, and the organism is identified to be susceptible to biofilm-relevant antibiotics (Darouiche, 2004; Konig et al., 2001; Trampuz and Widmer, 2006; Trebse et al., 2005; Widmer et al., 1992; Zimmerli et al., 1998). In a previous prospective study, in which 87 patients with orthopaedic implant-related infections were identified, $28 \%$ of the patients met the inclusion criteria for treatment with retention of the implant (including hip, knee, and ankle prostheses as well as internal fracture fixation devices), which resulted in a probability of survival without treatment failure of $86 \%$ at three years post-revision (Trebse et al., 2005). However, retaining a device that may be contaminated with biofilm can pose an increased challenge for treatment. The clinical algorithms typically recommend multiple-staged surgical interventions, beginning with meticulous debridement of the infected bone and soft tissue, coupled with localised and systemic antibiotics to treat the infection before bone reconstructive procedures can be pursued. Local antibiotic administration has the advantage of augmenting the systemic therapy by improving the antibiotic bioavailability directly at the infection site (Darouiche, 2004; Mihailescu et al., 2014; Tang et al., 2013; Valour et al., 2014; Zimmerli et al., 2004). Antibiotics are typically delivered locally using poly (methyl methacrylate) (PMMA) bone cement spacers (Darouiche, 2004; Jaeblon, 2010). However, PMMA spacers are compatible with only a limited number of antibiotics and have poor release profiles that affect the drug's bioavailability.

Rifampin is a critical antibiotic for management of Staphylococci in biofilm (Mihailescu et al., 2014; Tang et al., 2013), especially in cases where the implant is retained (Darouiche, 2004; Zimmerli et al., 2004). Unfortunately, 
PMMA spacers are not suitable for localised delivery of rifampin because this antibiotic impairs the polymerisation process by acting as a free radical scavenger (Beeching et al., 1986). Alternative biomaterials for local rifampin delivery, which include polymers (O'hara and Hickey, 2000; Waeiss et al., 2014), ceramics (Molina-Manso et al., 2012) and composites (Ahola et al., 2012; Gu et al., 2012), have been investigated in vitro, but the potential therapeutic efficacy of these rifampin-laden biomaterials has not yet been demonstrated in vivo. Furthermore, the in vivo efficacy of local rifampin delivery has previously been evaluated only as a prophylactic implant coating (Darouiche et al., 2007), but not as a treatment for an established bone infection with biofilm involvement.

Calcium phosphates $(\mathrm{CaP})$ are a class of ceramics that have been investigated as regenerative scaffolds for hard tissues and as drug delivery materials in orthopaedic and dental applications (Bose and Tarafder, 2011; Ginebra et al., 2006). CaP are amenable to additive fabrication technologies such as three-dimensional (3D) printing based on the concept of selective granular fusion or binding, which can enable the creation of medical imaging-guided, patient-specific bone substitutes (Klammert et al., 2010), and potentially the incorporation of drugs and bioactive factors. However, sintering of $3 \mathrm{D}$ printed $\mathrm{CaP}$ materials at ultra-high temperatures, which might be required to increase their strength, can obliterate the bioactivity of incorporated drugs. In contrast, low temperature 3D printing of $\mathrm{CaP}$ allows for fine spatial distribution of "bioink" (drug) within the printed geometry (Vorndran et al., 2010). Vorndran and colleagues demonstrated direct addition of vancomycin, an important antibiotic against methicillin-resistant $S$. aureus (MRSA), into CaP during the low temperature $3 \mathrm{D}$ printing process, which utilised a highly acidic binder (20 wt $\%$ phosphoric acid) (Vorndran et al., 2010). However, while the Vorndran study demonstrated a proof of concept, we have recently shown that the highly acidic binding solution results in residual acidity that can be highly cytotoxic (Inzana et al., 2014), and can significantly degrade the bactericidal activity of the incorporated drug.

In this study, we test the hypothesis that utilising a low acidity binder solution would enable low-temperature 3D printing of vancomycin- and rifampin-laden CPS while maintaining the bactericidal activity; and that these dualantibiotic CPS would significantly reduce the bacterial burden and osteolytic bone loss of an established implantassociated bone infection compared with vancomycinladen PMMA, which is considered a clinical standard of care. This hypothesis was studied using a previously developed mouse model of established implant-associated bone infection (Inzana et al., 2015). Prior to treatment, this murine model of established bone infection mimics the salient aspects of the challenging clinical conditions of implant-related osteomyelitis, including biofilm and bone loss. This mouse model was designed to simulate the clinical algorithms for implant retention, as described above, considering that a revision surgery takes place 7 days post-infection, the fixation plate remains mechanically stable, and an extensive debridement of both the bone and soft tissue is performed, along with a local and systemic antimicrobial treatment, as previously described (Inzana et al., 2015).

\section{Materials and Methods}

\section{Preparation of 3D printed antibiotic-laden CPS and PMMA materials}

A modified ZPrinter ${ }^{\circledR} 450$ (3D Systems; Andover, MA) was used to 3D print the CPS materials as previously described (Inzana et al., 2014). In this method, a phosphoric acidbased solution is selectively sprayed using inkjets that raster over a layered bed of calcium phosphate powder to initiate a dissolution-precipitation reaction, which forms 3D CPS after repeated layer-by-layer binding (Gbureck et al., 2007b). The calcium phosphate powder was $99 \%$ phase pure $\alpha$-tricalcium phosphate ( $\alpha$-TCP; InnoTERE $\mathrm{GmbH}$; Radebeul, Germany). The powder was bound during the $3 \mathrm{D}$ printing process by delivering a solution of $8.75 \mathrm{wt} \%$ phosphoric acid $+0.25 \mathrm{wt} \%$ Tween 80 from the inkjets, which was previously demonstrated to produce cytocompatible and osteoconductive scaffolds (Inzana et al., 2014). The powder layer thickness was set to $89 \mu \mathrm{m}$ and the binder liquid-to-powder ratio was set as 0.46 in the ZPrint ${ }^{\mathrm{TM}}$ software. The CPS materials were supplemented with antibiotics by either mixing vancomycin (Alfa Aesar; Ward Hill, MA) or rifampin (Sigma-Aldrich; St. Louis, MO) powder into the $\alpha$-TCP or by infusing an antibiotic solution into the CPS using a second set of 'colour' inkjets. Vancomycin was mixed into the powder to achieve a concentration of $10 \mathrm{wt} \%$, which yielded CPS with $5.2 \mathrm{wt} \%$ vancomycin $\left(\sim 365 \mu \mathrm{g} / \mathrm{CPS}\right.$; Vanco ${ }_{5 \%}$-CPS $)$. Rifampin was mixed into the powder substrate at a concentration of $1 \mathrm{wt} \%$ to yield CPS with $0.5 \mathrm{wt} \%$ rifampin $\left(\sim 35 \mu \mathrm{g} / \mathrm{CPS}\right.$; Vanco V $_{\%}+\mathrm{Rif}_{0.5 \%}$-CPS or $\mathrm{Rif}_{0.5 \%}$-CPS $)$. Alternatively, a solution of $10 \mathrm{mg} / \mathrm{mL}$ rifampin dissolved in $50 \% \mathrm{DMSO} / \mathrm{H}_{2} \mathrm{O} \mathrm{vol} / \mathrm{vol}$ was delivered using the inkjets to achieve a concentration of $0.05 \mathrm{wt} \%$ rifampin $\left(\sim 3.5 \mu \mathrm{g} / \mathrm{CPS} ;\right.$ Vanco $_{5 \%}+$ Rif $_{0.05 \%}$-CPS or $\mathrm{Rif}_{0.05 \%}$-CPS $)$. To create a sustained antibiotic release formulation, Vanco $_{5 \%}+$ Rif $_{0.5 \%}$-CPS were coated with poly (D,L-lactideco-glycolide) (PLGA; Vanco ${ }_{5 \%}+\mathrm{Rif}_{0.5} \%$-CPS/PLGA). The PLGA (50:50 L:G, MW 24-38 kDa; Sigma-Aldrich; St. Louis, MO) was dissolved into dichloromethane at a concentration of $5,12.5$, or $20 \mathrm{wt} \%$ in combination with $20 \mathrm{mg} / \mathrm{mL}$ rifampin. The CPS were soaked in this solution for $1 \mathrm{~h}$ to adsorb the rifampin-laden PLGA and were then dried at $40^{\circ} \mathrm{C}$ in a vacuum chamber overnight to evaporate the volatile solvent. The CPS/PLGA composites that were implanted into mice used $12.5 \mathrm{wt} \%$ PLGA. PMMA bone cement (SmartSet ${ }^{\mathbb{R}}$ MV Bone Cement; DePuy Synthes, Warsaw, IN) samples were prepared by hand mixing according to the manufacturer's instructions as a placebo (PBO-PMMA) or mixed with $5 \mathrm{wt} \%$ vancomycin $(0.5 \mathrm{mg}$ per murine spacer; Vanco ${ }_{5}$-PMMA) and formed in a custom mould.

\section{Characterisation of antibiotic release and functionality from CPS and PMMA materials}

The total antibiotic loaded into the $3 \mathrm{D}$ printed CPS was measured by dissolving scaffolds in $3 \mathrm{M} \mathrm{HCl}$ and 
measuring the concentration of vancomycin and rifampin ( $n=6$ samples/group) by optical absorbance at $280 \mathrm{~nm}$ using a spectrophotometer (Synergy Mx Microplate Reader, BioTek Instruments Inc.; Winooski, VT). The limit of quantification at $280 \mathrm{~nm}$ was $7 \mu \mathrm{g} / \mathrm{mL}$ for vancomycin and $3 \mu \mathrm{g} / \mathrm{mL}$ for rifampin.

Antibiotic functionality was measured through a KirbyBauer disc diffusion assay ( $n=6$ samples/group measured in duplicate). Vancomycin and rifampin were eluted into PBS from CPS or PMMA samples and the concentrations were measured by optical absorbance at $280 \mathrm{~nm}$ after passing the eluate through a $0.2 \mu \mathrm{m}$ filter. Fresh antibiotic solutions that spanned the concentrations of interest were used to generate a linear standard curve. Filter paper discs ( $7 \mathrm{~mm}$ diameter) were placed into $1 \mathrm{~mL}$ of each solution overnight at $4^{\circ} \mathrm{C}$ to adsorb the antibiotics. The discs were dabbed dry and then placed onto Luria agar plates, which were inoculated with a bioluminescent strain of methicillinsensitive $S$. aureus (Xen36; PerkinElmer, Inc.; Waltham, $\mathrm{MA})$, and the plates were incubated overnight at $37^{\circ} \mathrm{C}$. The diameters of the bacterial inhibition zones surrounding each disc were measured and the active antibiotic concentration was determined by comparing to the linear standard curve as previously described (Bonev et al., 2008). Statistically significant differences in the active antibiotic concentration were assessed by comparing to the non-printed control antibiotic solution through one-way ANOVA with Tukey's test for multiple comparisons.

To measure the antibiotic release kinetics, $1.8 \mathrm{~mm}$ diameter $\times 3 \mathrm{~mm}$ murine femoral scaffolds were $3 \mathrm{D}$ printed with either vancomycin or rifampin independently $(n=6$ samples/group). The cylinders were placed into sealed vials with $0.5 \mathrm{~mL}$ of PBS in static conditions at $37^{\circ} \mathrm{C}$. The cylinders were transferred to new vials with fresh PBS after $1,2,4,8$ and $24 \mathrm{~h}$ and then daily for up to 2 weeks. The vancomycin or rifampin concentrations were measured by optical absorbance at $280 \mathrm{~nm}$.

\section{Biomechanical properties of 3D printed antibiotic- laden CPS materials}

The flexural properties of the 3D printed CPS were assessed by 3 point bending to determine if supplementing these materials with antibiotics affects the mechanical strength ( $n=6-10$ /group). Samples were 3D printed as $4 \times 1 \times 14 \mathrm{~mm}$ bars and tested on a $10 \mathrm{~mm}$ support span using an Instron 8841 DynaMight ${ }^{\mathrm{TM}}$ Axial Testing System (Instron Corp.; Canton, MA) with a $50 \mathrm{~N}$ load cell. Flexural tests were performed at a displacement rate of $1 \mathrm{~mm} / \mathrm{min}$ until failure.

\section{Animals and surgical procedures}

All animal studies were performed in accordance with protocols approved by the University of Rochester's Committee on Animal Resources. Female BALB/cJ mice were purchased from Jackson Research Labs (Bar Harbor, ME) at 13-15 weeks of age and acclimated for one week prior to surgery. The surgeries were performed according to a previously described model (Inzana et al., 2015). Briefly, the right femur was exposed by a direct lateral approach and a 6-hole radiolucent polyether ether ketone (PEEK) plate with a $40 \mathrm{~nm}$ titanium coating was installed across the anterolateral surface using 4 titanium screws (RISystem; Davos, Switzerland). To prevent septic loosening from osteolysis around the screws, the fixation was reinforced in a cerclage-fashion by tying a 5-0 nylon monofilament suture around the bone and plate in between each pair of proximal and distal screws. A $0.7 \mathrm{~mm}$ transverse osteotomy was cut through the femoral mid-diaphysis using a $0.67 \mathrm{~mm}$ wire Gigli saw and a cutting guide (RISystem; Davos, Switzerland). A $1 \mathrm{~mm}$ radius semi-circle of fibrillar collagen sheet (Kensey Nash; Exton, PA) that was soaked for at least $2 \mathrm{~h}$ in an overnight culture of bioluminescent methicillin-sensitive $S$. aureus (Xen36; PerkinElmer, Inc.; Waltham, MA) was placed into the defect. Xen36 was modified from the parental strain ATCC 49525, which is a clinical isolate from a bacteraemia patient, to contain the lux $A B C D E$ operon. Xen36 was previously found to be a good candidate for longitudinal imaging in murine models of chronic implant-associated osteomyelitis (Pribaz et al., 2012) and was confirmed to form robust biofilms in vivo on the plates and screws of this murine model (Inzana et $a l ., 2015)$. The viable bacterial load on each collagen sheet was previously determined to be at least $8.0 \pm 2.9 \times 10^{4}$ colony forming units (CFU) (Inzana et al., 2015). The muscle and skin were closed with 5-0 nylon monofilament sutures and the infection was allowed to establish for 7 days until the revision surgery (Fig. 3a). At the time of revision, the infected tissue was debrided and the bone defect was widened to $3 \mathrm{~mm}$ to place a $1.8 \mathrm{~mm}$ diameter $\times 3 \mathrm{~mm}$ antibiotic CPS or PMMA spacer into the defect $(n=8-15$ / group; Fig. 3b). One mouse from the $\mathrm{Vanco}_{5}$-PMMA group was over-infected during the inoculation procedure and was therefore excluded, resulting in $n=7$ for this group. The muscle and skin were closed and the mice were allowed to heal for an additional 21 days. Select groups also received systemic vancomycin beginning at the revision surgery $(110 \mathrm{mg} / \mathrm{kg}$ twice daily subcutaneously (SC Vanco)) until sacrifice. Upon tissue harvest, the bone, soft tissue, fixation hardware, and antibiotic implants were placed into separate vials with $1 \mathrm{~mL}$ of sterile PBS and frozen at $-80^{\circ} \mathrm{C}$ for $\mathrm{CFU}$ analysis.

\section{Bioluminescent imaging of the active bacterial infection}

The Xen36 bacterial infection was evaluated longitudinally through bioluminescent imaging (BLI) using an IVIS Spectrum imaging system (PerkinElmer, Inc.; Waltham, MA) with a five-min dorsal exposure. The average luminescent radiance was quantified through the Living Image software (PerkinElmer, Inc.) by using a fixed-size region of interest (ROI) around the right thigh of each mouse. The area under the radiance curve post-revision surgery (Post-Rev Radiance AUC) was computed to quantify the pathogenic burden of the infection.

\section{Radiographic imaging and analysis of osteolysis and reactive bone formation}

To monitor changes to the bone, X-rays (LX-60 X-ray Cabinet, Faxitron Bioptics LLC; Tucson, AZ) were taken at $26 \mathrm{kV}$ with an exposure time of $5 \mathrm{~s}$. One day after the revision surgery (day 8 ) and on the final day of the study (day 28), the right femur of each mouse was scanned in 
vivo by micro-computed tomography (micro-CT; VivaCT 40, Scanco Medical; Bassersdorf, Switzerland) with a $17.5 \mu \mathrm{m}$ isotropic voxel size using an integration time of $300 \mathrm{~ms}$, energy of $55 \mathrm{kV}$, and intensity of $145 \mu \mathrm{A}$. 3D reconstruction and quantitative analysis of the micro-CT images was performed using Amira software (Amira 5.4.5, FEI Visualization Sciences Group; Burlington, MA). For each mouse, micro-CT images that were collected on day 8 were co-registered with images collected on day 28 to measure the volumetric changes to the bone. The proximal ROI and distal ROI encompassed the bone from the edge of the $3 \mathrm{~mm}$ osteotomy to the nearest screw on the proximal and distal sides of the defect, respectively (Fig. 6a). The images were binarised using a global bone mineral density threshold of $435 \mathrm{mg} \mathrm{HA} / \mathrm{cm}^{3}$ to measure the volumetric bone changes (reactive bone formation as well as osteolytic resorption), which were normalised to the original bone volume at day 8 in each ROI.

\section{Scanning electron microscopy (SEM) imaging of the implants}

The plate and screws from each mouse were fixed in $2.5 \%$ glutaraldehyde $/ 4 \%$ paraformaldehyde in $0.1 \mathrm{M}$ Cacodylate buffer, post-fixed in $1 \%$ osmium tetroxide, dehydrated, and gold sputter coated. The samples were imaged by SEM (LEO 982 FE-SEM, Carl Zeiss SMT; Thornwood, NY) for qualitative assessment of bacterial colonisation and biofilm formation on the implant surfaces.

\section{Measurement of bacterial colonisation}

To measure the viable bacterial load remaining in the bone and soft tissue, each sample was thawed and homogenised in $3 \mathrm{~mL}$ of sterile PBS. Residual bacteria were dislodged from the fixation hardware and antibiotic implants by sonicating the samples in $1 \mathrm{~mL}$ sterile PBS for $3 \mathrm{~min}$ at 35 kHz (VWR Symphony Ultrasonic Cleaner; VWR Intl.; Radnor, PA) and then vortexed. The tissue homogenates and sonicated suspensions were spread on Luria agar plates in 10 -fold dilutions and incubated overnight at $37^{\circ} \mathrm{C}$ for CFU counts, which were normalised to the mass of each sample. CFU data were transformed using $\log _{10}(1+\mathrm{X})$ to achieve normal distributions that retain zero values.

\section{Measurement of functional antibiotics from retrieved spacers}

Following sonication of the antibiotic implants that were retrieved after 21 days of implantation in the mice, the CPS and PMMA spacers were vortexed in $1 \mathrm{~mL}$ PBS to elute any residual antibiotics. Two filter paper discs ( $7 \mathrm{~mm}$ diameter) were placed into each vial of eluate overnight at $4^{\circ} \mathrm{C}$. The discs were placed onto Xen36 inoculated Luria agar plates and incubated for $12 \mathrm{~h}$ at $37^{\circ} \mathrm{C}$. The plates were imaged by BLI to measure the bacterial viability by placing a $7 \mathrm{~mm}$ diameter region of interest around each disc.

\section{Data analysis}

Two in vivo studies were performed. First, Vanco $_{5 \%}$-PMMA was compared against Vanco $_{5 \%}+$ Rif $_{0.05 \%}$-CPS, with or without SC Vanco, in a two-way ANOVA with Sidak's test for multiple comparisons when the interactive term was significant. Second, Vanco ${ }_{5 \%}$-PMMA was compared with the lower rifampin dose $\left(\right.$ Vanco $_{5 \%}+$ Rif $\left._{005 \%}-\mathrm{CPS}\right)$, the higher rifampin dose $\left(\right.$ Vanco $_{5 \%}+\mathrm{Rif}_{0.5}$-CPS), and the sustained release formulation (Vanco ${ }_{5 \%}+\mathrm{Rif}_{0.5 \%}$-CPS/ PLGA), all with concomitant SC Vanco, using ANOVA with Dunnett's post-hoc test for multiple comparisons against Vanco $_{5 \%}$-PMMA if the ANOVA was significant. Data were confirmed to satisfy assumptions of a Gaussian distribution based on the D'Agostino-Pearson normality test or a nonparametric alternative was used where appropriate. Considering the lack of a nonparametric alternative for two-way ANOVA and the bimodal distribution of CFU data (due to culture negative samples), Fischer's exact test was also performed to complement the two-way ANOVA tests of the CFU data. The $2 \times 2$ contingency table included mice that received $\mathrm{Vanco}_{5 \%}-\mathrm{PMMA}$ and those that received Vanco $_{5 \%}+$ Rif $_{0.05 \%}$-CPS local treatment, regardless of whether SC Vanco was also administered. The lowest CFU measurement in the Vanco $_{5}$-PMMA group was set as the threshold for binarising the outcome data. For assays where a culture negative sample was observed in the Vanco $_{5 \%}$-PMMA group, the data was analysed based on the number of culture negative samples. Bonferroni's correction was applied to control the family-wise error rate for the multiple ANOVAs conducted at each time point over the BLI time courses. Differences were considered significant for multiplicity-adjusted $p<0.05$. All $p$ values presented are multiplicity-adjusted.

\section{Results}

\section{D printing and physical characterisation of antibiotic-laden CPS}

Antibiotic-laden CPS were 3D printed using the previously optimised lower acidity binding solution (8.75\% phosphoric acid $+0.25 \%$ Tween 80 ) (Inzana et al., 2014). The 3D printed CPS had a coarser surface morphology and increased porosity compared to PMMA spacers that are typically used in the management of osteomyelitis (Fig. 1). The bioactivity of the antibiotics within the low acidity 3D printed CPS was retained, whether vancomycin in solution was infused using the 'colour' inkjets $(82 \% \pm 2 \%$ bioactive; Fig. 2a) or vancomycin powder was mixed into the $\alpha$-TCP powder (70 \% $\pm 11 \%$ bioactive; Fig. $2 b)$. In contrast, a highly acidic ( $20 \mathrm{wt} \%$ phosphoric acid) binding solution significantly degraded the bactericidal activity of the printed vancomycin $(30 \% \pm 9 \%$ active; $p<0.0001$; Fig. 2a). Since PMMA tends to sequester the majority of impregnated antibiotic, the 3D printed CPS delivered over 6-fold more functional vancomycin than equivalently loaded PMMA(Fig. 2c). Further, the addition of antibiotics did not compromise the mechanical strength of the CPS materials (Table 1). Rifampin also retained 35 to $50 \%$ of its bactericidal activity when mixed into the $\alpha$-TCP powder $(0.5 \mathrm{wt} \%)$ or infused using the inkjets $(0.05 \mathrm{wt} \%)$ and $3 \mathrm{D}$ printed with the lower-acidity binder solution (Fig. 2d). In all subsequent in vivo experiments, rifampin was always delivered in combination with vancomycin, because the bacteria can develop resistance to rifampin very rapidly when it is used as a monotherapy (Vergidis et al., 2011; Zimmerli et al., 2004). 

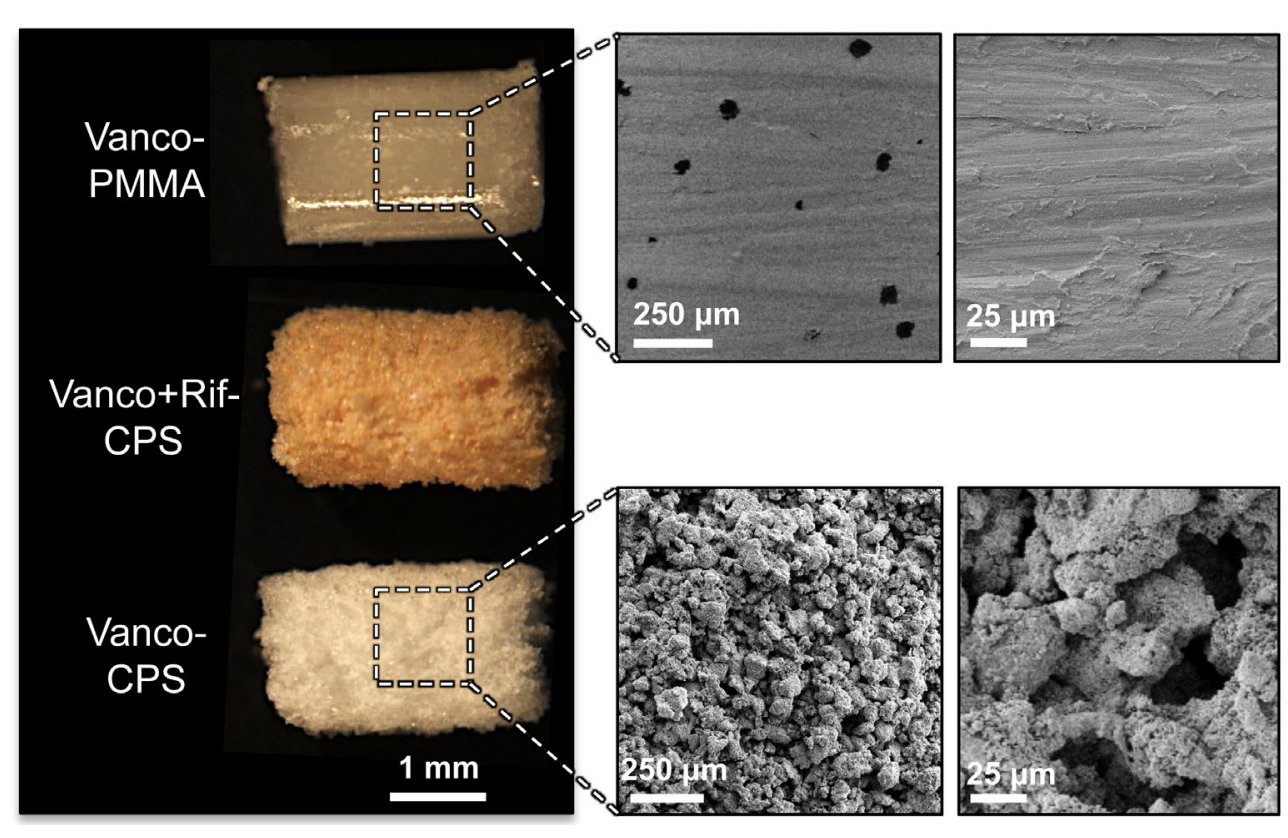

Fig. 1. Differences in the surface morphology and porosity between $3 \mathrm{D}$ printed CPS and PMMA spacers. Macroscopic and microscopic (SEM) images of $3 \mathrm{D}$ printed CaP scaffolds loaded with vancomycin and rifampin compared to a manually moulded PMMA spacer mixed with vancomycin.

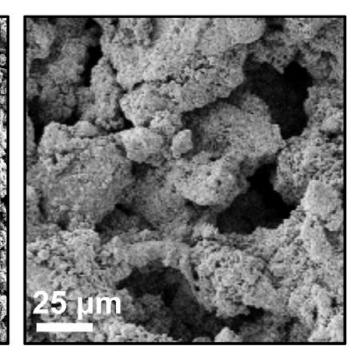

a

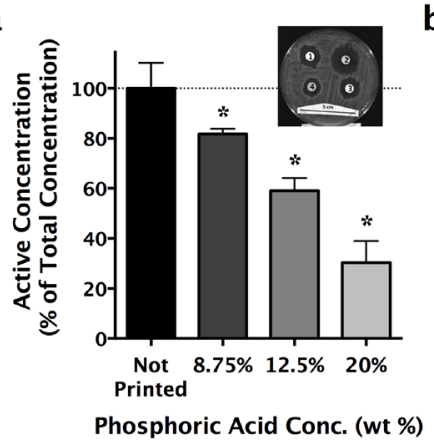

b

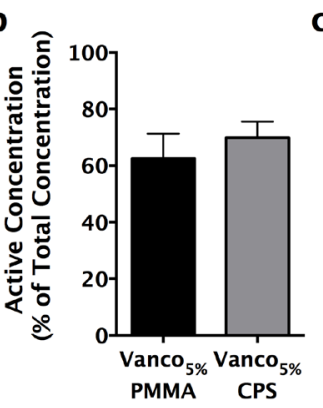

C

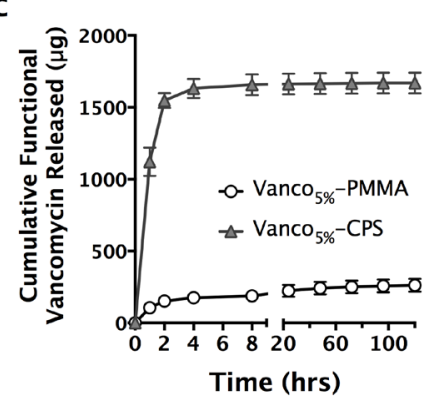

d

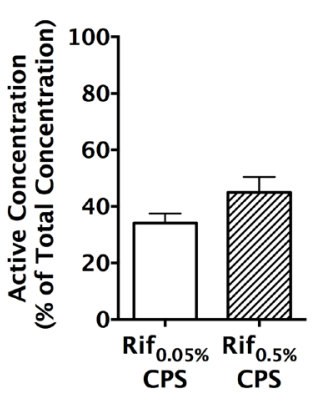

Fig. 2. Antibiotics retain their bactericidal activity after $3 \mathrm{D}$ printing into calcium phosphate scaffolds (CPS). Bactericidal activity was determined by the size of the bacterial inhibition zone in Kirby-Bauer disc diffusion assays of antibiotic eluate delivered using filter paper discs (inset in a), compared against linear standards. (a) Vancomycin retains up to $80 \%$ bactericidal activity when infused into 3D printed CPS using the inkjets in parallel with an $8.75 \mathrm{wt} \%$ phosphoric acid binding solution. *denotes $p<0.05$ vs. not-printed, by Tukey's test after ANOVA $(n=6 /$ group). (b) Bactericidal activity of vancomycin is also retained when the antibiotic is mixed into the calcium phosphate powder phase (Vanco ${ }_{5 \%}$-CPS) and 3D printed with $8.75 \mathrm{wt} \%$ phosphoric acid $+0.25 \%$ Tween 80 as the binder solution. (c) Vanco $_{5 \%}$-CPS released over 6-fold more functional vancomycin than loaded PMMA (Vanco ${ }_{5 \%}$-PMMA). (d) Rifampin bactericidal activity is also retained with this binder solution when the CPS are supplemented with rifampin using the inkjets ( Rif $_{0.05 \%}$-CPS) or by mixing into the powder phase ( Rif $\left._{0.5 \%}-\mathrm{CPS}\right)$. Data presented as mean \pm standard error of the mean; $n=6$ /group).

Table 1. Flexural properties of antibiotic-laden CPS

\begin{tabular}{|c|c|c|c|}
\hline & Maximum Stress (kPa) & Elastic Modulus (MPa) & Toughness $\left(\mathrm{mJ} / \mathrm{cm}^{3}\right)$ \\
\hline CPS & $63.0 \pm 4.8$ & $18.9 \pm 3.7$ & $0.12 \pm 0.03$ \\
\hline Vanco $_{50}-\mathrm{CPS}$ & $66.7 \pm 8.9$ & $17.4 \pm 3.4$ & $0.18 \pm 0.05$ \\
\hline Vanco $_{50}+$ Rif $_{0050}-\mathrm{CPS}$ & $62.3 \pm 11.1$ & $16.6 \pm 4.0$ & $0.14 \pm 0.04$ \\
\hline Vanco $_{50}+$ Rif $_{050}-\mathrm{CPS}$ & $69.1 \pm 9.2$ & $19.1 \pm 4.0$ & $0.16 \pm 0.05$ \\
\hline Vanco $_{50}+$ Rif $_{050}-\mathrm{CPS} /$ PLGA & $3134 \pm 586$ & $721 \pm 90$ & $9.9 \pm 3.6$ \\
\hline
\end{tabular}

Flexural strength was measured by 3-point bending with $1 \times 4 \times 14 \mathrm{~mm}$ bars on a $10 \mathrm{~mm}$ support span at a displacement rate of $1 \mathrm{~mm} / \mathrm{min}$. Values are represented as means and standard deviation. $n \geq 6$ per group. 
a

b

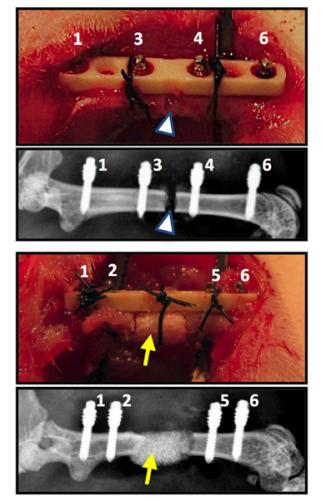

d

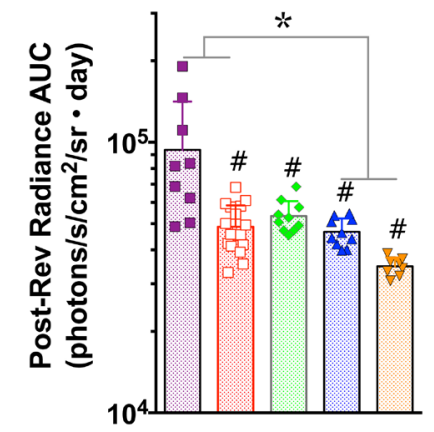

c

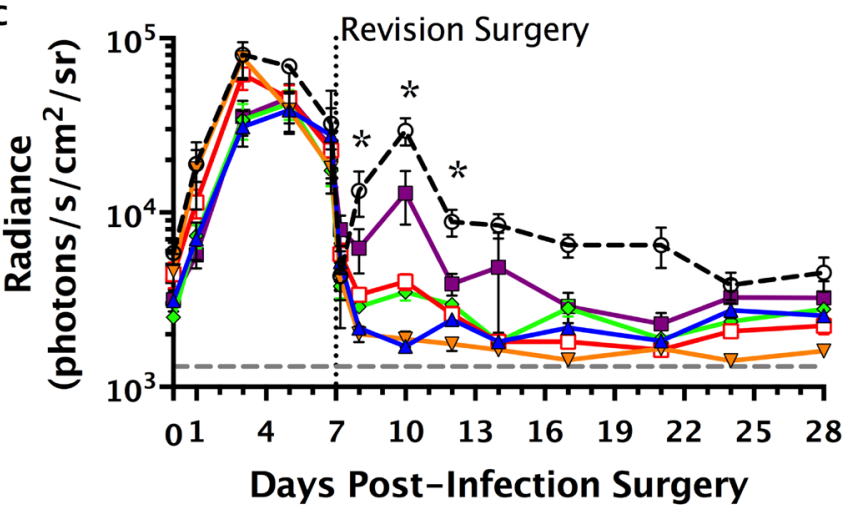

Fig. 3. Low dose local antibiotic delivery using 3D printed CPS reduced the bacterial burden after revision. (a) A fullthickness femoral osteotomy (to simulate a fracture) is stabilised with a fixation plate and screws and then infected using a collagen sheet (white triangle) inoculated with a bioluminescent strain of methicillin-sensitive S. aureus (Xen36). (b) Seven days after the initial osteotomy and the establishment of infection, an implant-retaining revision surgery is performed, in which the infected tissue is debrided, infected screws 3 and 4 are replaced with sterile screws 2 and 5 , and an antibiotic-laden spacer (yellow arrow) is placed within the critical bone void. (c) Local antibiotic treatment with $\mathrm{Vanco}_{5 \%}+\mathrm{Rif}_{0.05 \%}$-CPS significantly reduced the bacterial load compared with Vanco ${ }_{5 \%}-$ PMMA, independent of subcutaneous vancomycin ( $\mathrm{SC}$ Vanco) administration as observed through longitudinal bioluminescent imaging (BLI). The area under the radiance curve (AUC) following revision surgery further demonstrates the reductions in bacterial metabolic load with each treatment (d). *denotes multiplicity-adjusted $p<0.05$ for the effect of Vanco $_{5 \%}+\mathrm{Rif}_{0.05 \%}{ }^{-} \mathrm{CPS}$ $v s$. Vanco ${ }_{5 \%}$-PMMA by 2-way ANOVA. " denotes multiplicity-adjusted $p<0.05 v s$. Vanco ${ }_{5 \%}-$ PMMA after ANOVA with Dunnett's test for post-hoc comparisons. $n=7-15 /$ group.

In vivo efficacy of low dose rifampin- and vancomycin-laden CPS scaffolds

The pathogenic burden, which was measured longitudinally using BLI, was significantly reduced by the Vanco $_{5 \%}+$ Rif $_{0.05 \%}$-CPS treatment compared with Vanco $_{5 \%}$-PMMA on days 8-12 (1700 $\pm 240 v s .8770 \pm 4720$ photons $/ \mathrm{s} / \mathrm{cm}^{2} / \mathrm{sr}$, respectively at day $10 ; p=0.001$ by 2 -way ANOVA; Fig. 3c) and in the overall area under the post-op BLI curve $\left(41000 \pm 8000\right.$ vs. $64000 \pm 36000$ photons $/ \mathrm{s} / \mathrm{cm}^{2} /$ $\mathrm{sr} \times$ day; $p=0.0001$ by 2-way ANOVA; Fig. 3d). Further, only mice treated with $\mathrm{Vanco}_{5 \%}+\mathrm{Rif}_{0.05 \%}$-CPS local therapy had a significantly reduced BLI signal from Day 7 post-op through Day 10 (mean change of $-2957 \pm 2747$ photons/s/ $\mathrm{cm}^{2} / \mathrm{sr} ; p=0.0003$ by one sample $t$-test $v s$. zero; Fig. $3 \mathrm{c}$ ). For results regarding the effect of the local treatment strategy $\left(\right.$ Vanco $_{5 \%}+\mathrm{Rif}_{0.05 \%}-\mathrm{CPS}$ vs. Vanco ${ }_{5 \%}$-PMMA) from 2-way ANOVA, the mean and SD values and percent of culture negative samples reported in this section are pooled from the groups of each local treatment, irrespective of SC Vanco treatment. The Vanco ${ }_{5 \%}$-CPS treatment also significantly reduced the overall area under the post-op BLI curve relative to Vanco $_{5 \%}$-PMMA without SC Vanco $\left(53000 \pm 7000\right.$ vs. $94000 \pm 48000$ photons $/ \mathrm{s} / \mathrm{cm}^{2} / \mathrm{sr} \times$ day; $p=0.0003$ by ANOVA; Fig. $3 d$ ). Quantitative bacterial colonisation determined by CFU assays confirmed the findings from the longitudinal BLI. The bones (Fig. 4a) and soft tissues (Fig. 4b) from animals that received Vanco $_{5 \%}+$ Rif $_{0.05}$-CPS local treatment (regardless of SC Vanco) were bacteria culture negative in $19 \%(3 / 16)$ and $63 \%(10 / 16)$ of the mice, respectively, compared with $0 \%(0 / 22)$ and $5 \%(1 / 22)$ of the mice that received Vanco $_{5 \%}$-PMMA local treatment (regardless of SC Vanco) or $0 \%(0 / 8)$ and $0 \%(0 / 8)$ that received Vanco $_{5 \%}$-CPS. The importance of co-treatment with rifampin was demonstrated by the lesser efficacy of the vancomycin monotherapy and the lack of significant differences in CFU counts between $\mathrm{Vanco}_{5 \%}$-CPS and $\mathrm{Vanco}_{5 \%}$-PMMA. Vanco $_{5 \%}+\mathrm{Rif}_{0.05 \%}$-CPS treatment significantly reduced the bacterial load compared with Vanco $_{5 \%}$-PMMA on the antibiotic implants $(1.22 \pm 1.90$ vs. $2.78 \pm 1.94 \log$ $(1+\mathrm{CFU} / \mathrm{g})$, respectively; $p=0.013$ by 2 -way ANOVA; $p=0.020$ by Fischer's exact test; Fig. 4c). The fixation plate and screws were colonised in $100 \%$ of the mice and the bacteria colonising them may not have been 
a

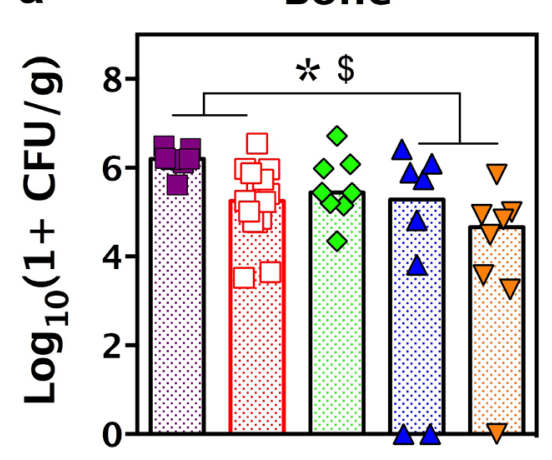

C Antibiotic Implant

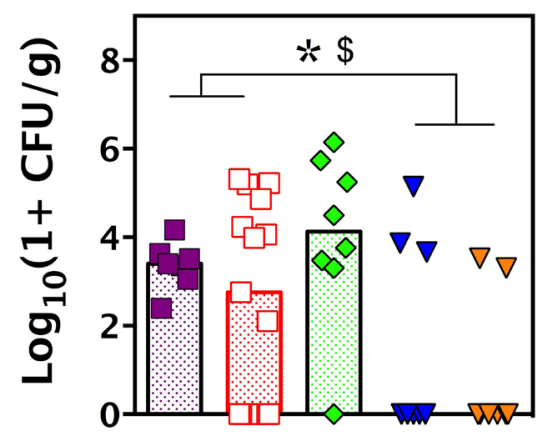

b

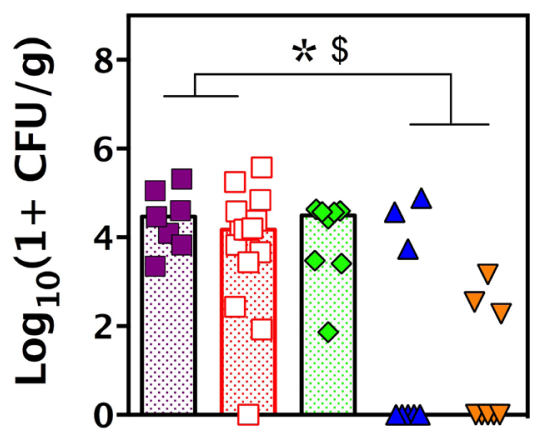

d Hardware

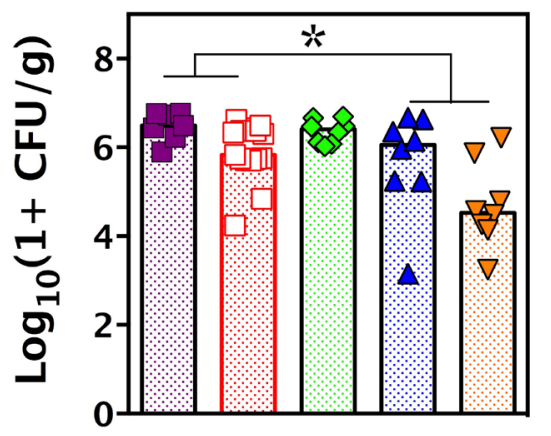
$\diamond \mathrm{Vanco}_{5 \%}$-CPS
\ Vanco $_{5 \%}+\mathrm{Rif}_{0.05 \%}$-CPS
- Vanco ${ }_{5 \%}$-PMMA
$\nabla$ Vanco $_{5 \%}+\mathrm{Rif}_{0.05 \%}$-CPS + SC Vanco
- Vanco $_{5 \%}$-PMMA + SC Vanco

Fig. 4. Bacterial colonisation, measured by end-point colony forming unit (CFU) assays, was reduced by low dose local antibiotic delivery using 3D printed CPS. (a) CFU quantification from the homogenised femoral bone, (b) soft tissue of the thigh, (c) the sonicated antibiotic implants, and (d) the fixation plate and screws. *denotes $p<0.05$ for the effect of Vanco $_{5 \%}+$ Rif $_{0.05 \%}$-CPS $v s$. Vanco ${ }_{5 \%}$-PMMA by 2-way ANOVA. ${ }^{\text {denotes }} p<0.05$ for the effect of Vanco $_{5 \%}+$ Rif $_{0.05}$-CPS $v s$. Vanco ${ }_{5 \%}$-PMMA by Fischer's exact test. Vanco ${ }_{5 \%}-$ CPS was not significantly different from Vanco ${ }_{5}$-PMMA by Dunn's test after nonparametric ANOVA (Kruskal-Wallis) in each tissue and implant. $n=7-15 /$ group. Bars indicate the median of each group.

significantly affected by the Vanco ${ }_{5 \%}+\mathrm{Rif}_{0.05 \%}-\mathrm{CPS}$ local treatment compared with Vanco $_{5 \circ}-\mathrm{PMMA}(5.53 \pm 1.09 \mathrm{vs}$. $6.08 \pm 0.62 \log (1+\mathrm{CFU} / \mathrm{g}) ; p<0.001$ by 2 -way ANOVA; $p=0.072$ by Fischer's exact test; Fig. $4 d$ ). Biofilm on the plates and screws was confirmed through scanning electron micrographs (Fig. 5).

Local antibiotic treatment with Vanco $_{5 \%}+$ Rif $_{0.05 \%}-\mathrm{CPS}$ significantly reduced the osteolytic bone resorption compared with Vanco $_{5 \%}$-PMMA and Vanco $_{5}$-CPS ( $23 \pm 7 \%$ vs. $34 \pm 11 \%$ resorption, respectively; $p<0.0001$; Fig. 6b) and produced a significantly more positive net bone change ( $-8.9 \% \pm 12 \%$ vs. $-21 \% \pm 16 \%$, respectively; $p=0.0012$; Fig. 6d). No significant differences were observed in the volume of bone formation between all of the treatment groups (Fig. 6c). Radiographic changes to the bone were not significantly different between Vanco $_{5 \%}$-CPS and Vanco $_{5 \%}$-PMMA in all of the measurements.
In vivo efficacy of antibiotic-laden CPS with a higher rifampin dose and sustained release

Next, we explored whether the efficacy of rifampin- and vancomycin-laden CPS could be enhanced by increasing the rifampin dose 10 -fold to $0.5 \mathrm{wt} \%$ (Vanco $_{5 \%}+\mathrm{Rif}_{0.5 \%}$-CPS) or by coating the higher dose rifampin-loaded CPS with PLGA to extend the time course of antibiotic release (Vanco $\left._{5 \%}+\mathrm{Rif}_{0.5 \%}-\mathrm{CPS} / \mathrm{PLGA}\right)$. The PLGA coated designs displayed an initial burst release of rifampin $(51 \mu \mathrm{g}$ in the first $24 \mathrm{~h}, 56 \%$ of total released for $12.5 \mathrm{wt} \%$ PLGA), followed by a sustained release $(1.8 \pm 0.8 \mu \mathrm{g} /$ day after day 1 , excluding the secondary burst at days 8-9) for at least 2 weeks in vitro (Fig. 7a,b). The design of the PLGA coating for in vivo analysis was selected to be $12.5 \mathrm{wt} \%$ based on a similar burst release of rifampin during the first $24 \mathrm{~h}$ between the uncoated Vanco $_{5 \%}+\mathrm{Rif}_{0.5 \%}-\mathrm{CPS}$ and the Vanco $_{5 \%}+$ Rif $_{0.5 \%}-\mathrm{CPS} / \mathrm{PLGA}_{12.5 \%}$ groups (Fig. 7a). Over the two weeks of elution in vitro, all of the PLGA 

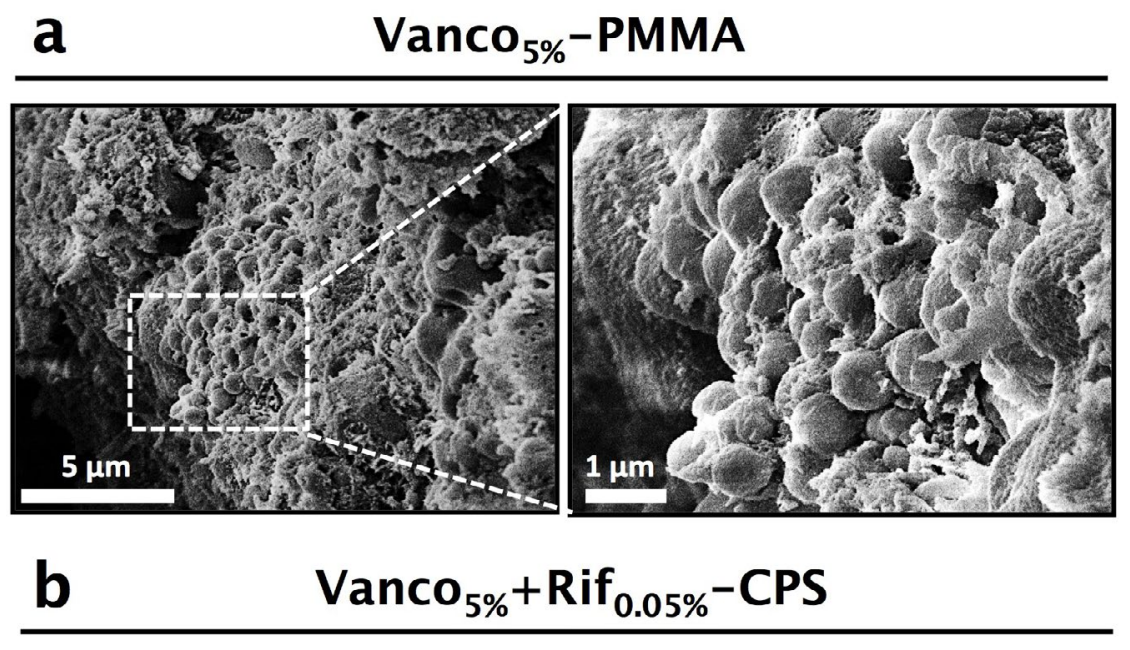

Fig. 5. Bacteria were observed embedded within biofilm on the plates and screws from all groups at day 28 of the study through scanning electron micrographs (SEM).
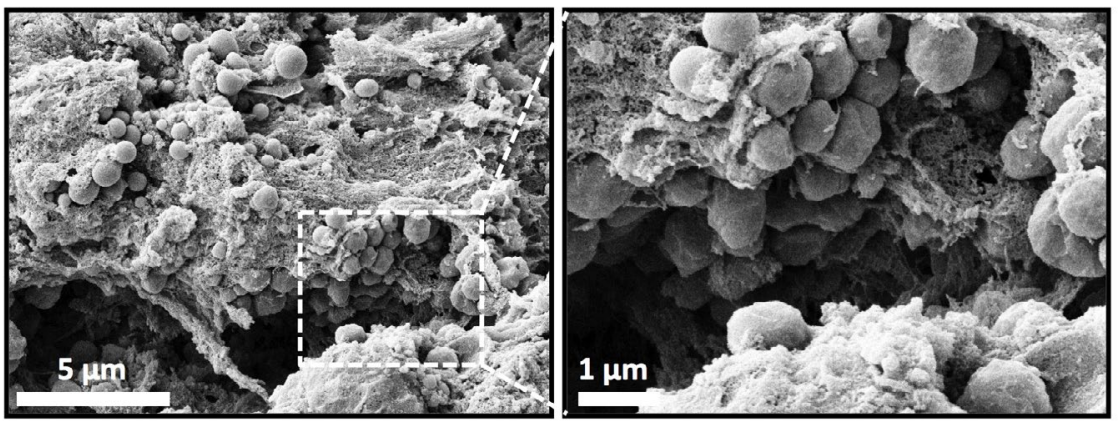

a

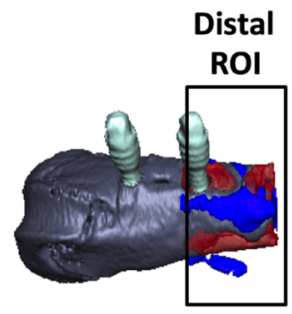

b

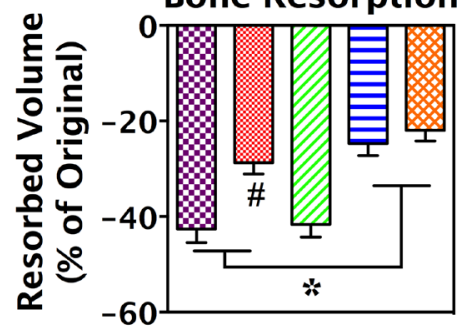

Wanco ${ }_{5 \%}$-PMMA
Vanco ${ }_{5 \%}$-PMMA + SC Vanco
C

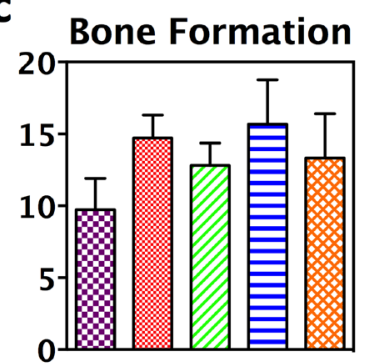

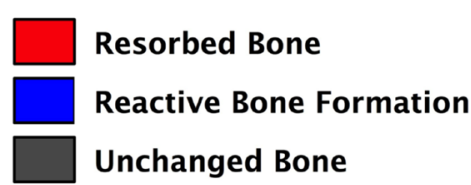

d

d Net Bone Change

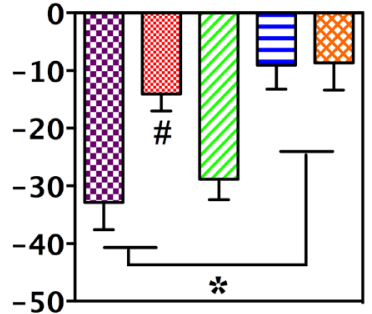

$$
\begin{aligned}
& \text { Vanco } \\
& \text { Vanco }_{5 \%}-\mathrm{CPS} \\
& \text { Vanco }_{5 \%}+\mathrm{Rif}_{0.05 \%}-\mathrm{CPS} \\
& \text { RaS }_{0.05} \text { SC Vanco }
\end{aligned}
$$

Fig. 6. Local rifampin therapy and systemic vancomycin significantly reduced the infection-induced osteolysis. (a) Micro-CT images collected on day 8 were co-registered with images collected on day 28 to measure the volumetric changes to the bone in two ROIs encompassing the osteotomy to the nearest screw on the proximal and distal sides of the defect, respectively, to measure the volumetric bone changes (osteolytic resorption (red) and reactive bone formation (blue)). Treatment with $\mathrm{Vanco}_{5 \%}+\mathrm{Rif}_{0.05 \%}$-CPS and SC Vanco each resulted in significantly reduced osteolytic bone resorption (b) and more positive net bone changes (d) after 3 weeks of treatment (day 28 of the study). The levels of reactive bone formation were not significantly different (c). $*$ denotes $p<0.05$ for the effect of Vanco $_{5 \%}+$ Rif $_{0.05 \%}-$ CPS $v s$. Vanco ${ }_{5 \%}$-PMMA by 2-way ANOVA. \#denotes $p<0.05$ for the effect of SC Vanco by Sidak's test after 2-way ANOVA with a significant interactive effect. Vanco $_{5 \%}$-CPS was not significantly different from Vanco ${ }_{5 \%}$-PMMA by Dunn's test after nonparametric ANOVA (Kruskal-Wallis) in each metric. $n=7-15 /$ group. 
a

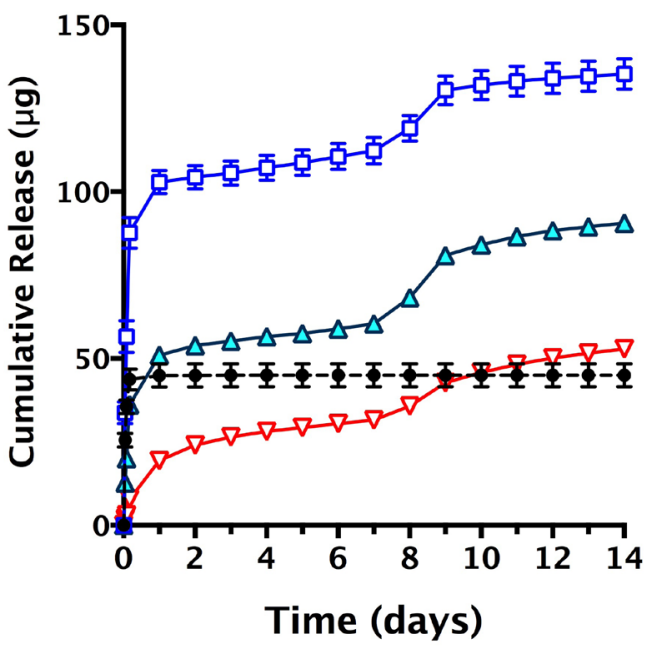

b

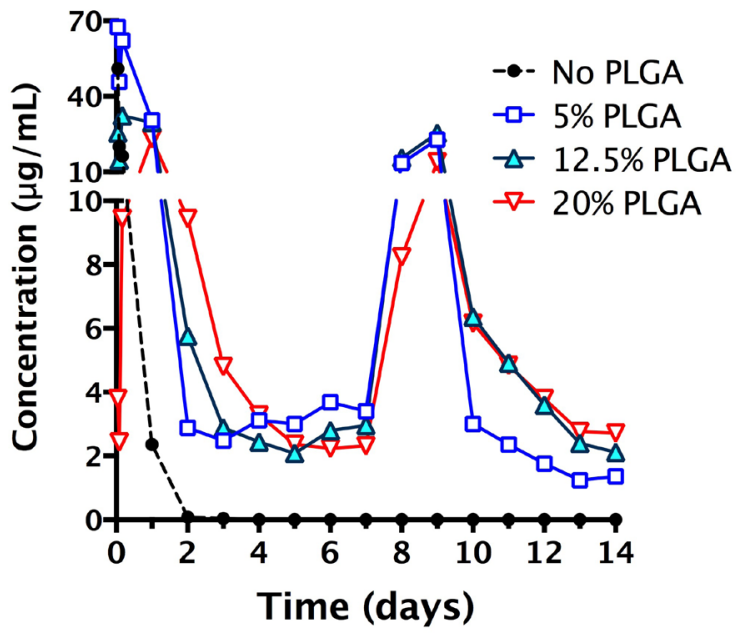

Fig. 7. Release kinetics of rifampin as a function of PLGA coating. (a) $\mathrm{Rif}_{05 \%}$-CPS that were coated by dipping in a $12.5 \%$ PLGA solution, supplemented with $20 \mathrm{mg} / \mathrm{mL}$ rifampin, released a similar amount of rifampin as uncoated $\mathrm{Rif}_{0.5 \%}$-CPS during the first $24 \mathrm{~h}$, followed by a sustained release of rifampin for at least 2 weeks $(n=5)$. The $\mathrm{Vanco}_{5 \%}+\mathrm{Rif}_{0.5 \%}$-CPS that were uncoated or coated with the $12.5 \%$ PLGA solution were studied in vivo. (b) The daily rifampin concentrations were well above the minimum inhibitory concentration (MIC; $0.08 \mu \mathrm{g} / \mathrm{mL}$ ) of rifampin-sensitive S. aureus for the full 2 weeks.

groups maintained daily rifampin concentrations that were well above the planktonic S. aureus MIC $(0.08 \mu \mathrm{g} /$ $\mathrm{mL}$ (Mihailescu et al., 2014)) (Fig. 7b). In addition, the PLGA coating significantly enhanced the flexural strength, stiffness, and toughness of the $3 \mathrm{D}$ printed $\mathrm{CaP}$ scaffolds (Table 1).

The in vivo efficacy of the higher rifampin concentration and sustained release formulations was examined alongside the original low dose design and compared to $\mathrm{Vanco}_{5 \%}{ }^{-}$ PMMA, with all of the groups receiving concomitant systemic vancomycin. None of the mice possessed gross pathological signs of infection at the end of the 3-week treatment time course. All of the rifampin-laden CPS treatments significantly improved the early infection
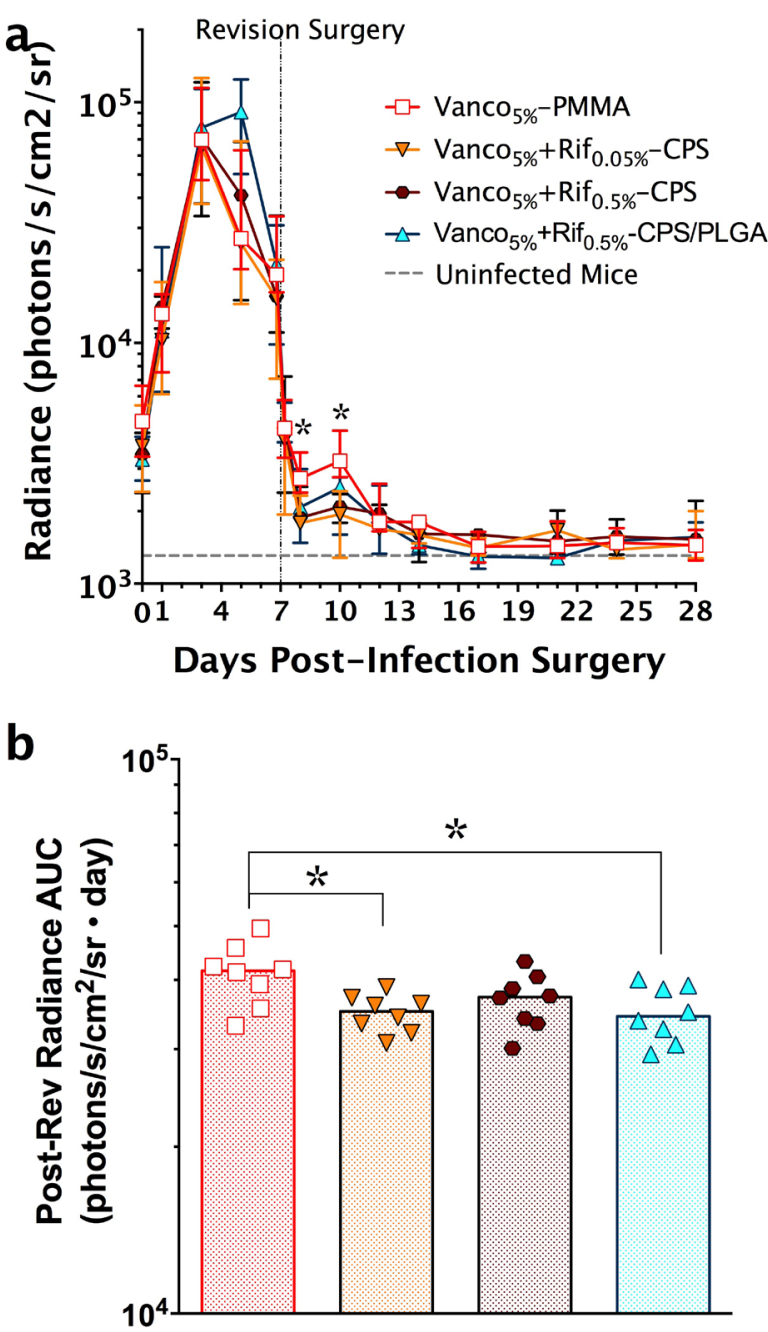

Fig. 8. The bactericidal efficacy of rifampin- and vancomycin-laden CPS is enhanced by higher rifampin dose and slow-release antibiotic delivery at 1 and 3 days after revision surgery compared with the Vanco $_{5 \%}$ - PMMA. (a) In vivo, the bacterial burden was significantly reduced at early time points (Days 8 and 10) after revision surgery by each of the rifampinladen CPS treatments compared to $\mathrm{Vanco}_{5 \%}$-PMMA, as determined by longitudinal bioluminescence. (b) The Vanco $_{5 \%}+$ Rif $_{0.05 \%}$-CPS and Vanco $_{5 \%}+$ Rif $_{0.5 \%}$-CPS/ PLGA treatments each reduced the post-revision radiance AUC compared to Vanco ${ }_{5 \%}$-PMMA spacers. All treated groups concomitantly received systemic vancomycin by subcutaneous injection. *denotes multiplicity-adjusted $p<0.05 v s$. Vanco ${ }_{5 \%}$ - PMMA by Dunn's test after nonparametric ANOVA (KruskalWallis). Bonferonni's correction was applied for analysis of the multiple time points across the bioluminescence time course. $n=8-15 /$ group. Bars and data points are medians and error bars are interquartile range.

management at 1 and 3 days after revision surgery compared with the Vanco $_{5 \%}$-PMMA $(2080 \pm 560$ vs. $3720 \pm 1400 \mathrm{photons} / \mathrm{s} / \mathrm{cm}^{2} / \mathrm{sr}$, respectively at 3 days postrevision; $p<0.001$; Fig. 8).

The bacterial colonisation of the bone, as measured by endpoint $\mathrm{CFU}$ assays, was significantly reduced by the 
a

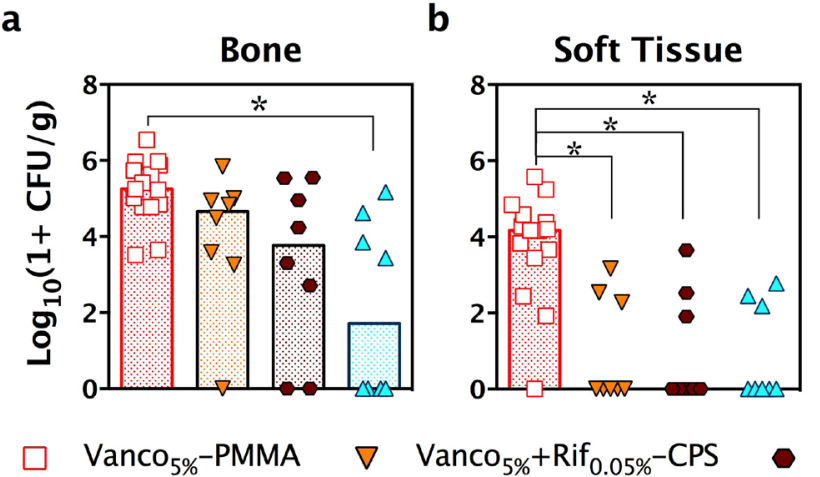

C

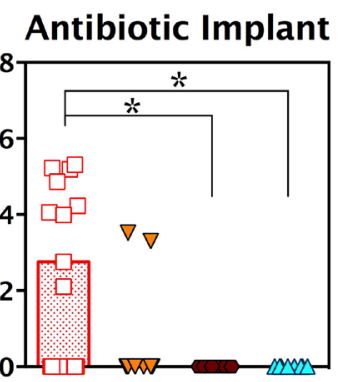

d Fixation Hardware

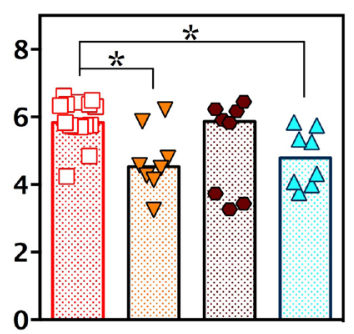

Fig. 9. Bacterial colonisation, measured by end-point colony-forming unit (CFU) assays after 3 weeks of treatment (day 28 of study), in the homogenised femoral bone (a) and soft tissue of the thigh (b) as well as on the sonicated antibiotic implants (c) and on the fixation plate and screws (d) was significantly reduced by local rifampin treatments compared to Vanco $_{5 \%}$-PMMA. All of the groups concomitantly received systemic vancomycin by subcutaneous injection. *denotes multiplicity-adjusted $p<0.05 v s$. Vanco ${ }_{5 \%}$-PMMA by Dunn's test after nonparametric ANOVA (Kruskal-Wallis). $n=8-15$ /group. Bars indicate the median of each group.

a
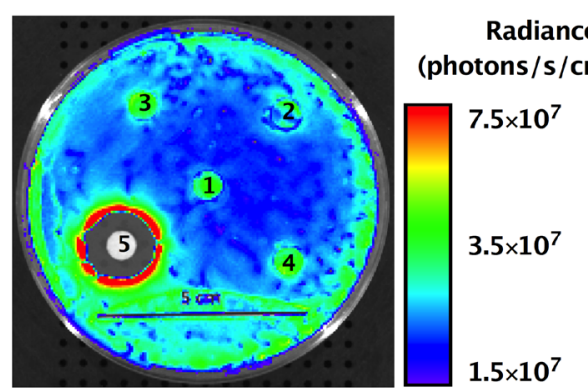

1. PBO-PMMA

2. VanCO $_{5 \%}-\mathrm{PMMA}$

3. Vanco ${ }_{5 \%}+$ Rif $_{0.05 \%}$-CPS

4. Vanco $_{5 \%}+$ Rif $_{0.5 \%}$-CPS

5. Vanco $_{5 \%}+$ Rif $_{0.5 \%}-$ CPS/PLGA b

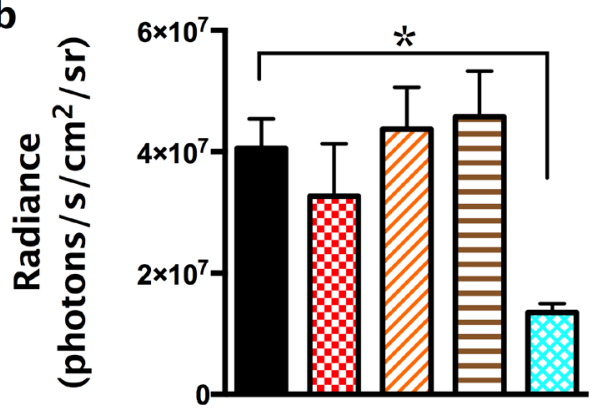

PBO-PMMA $\square$ Vanco $_{5 \%}+$ Rif $_{0.5 \%}$-CPS

D. Vanco ${ }_{5 \%}$-PMMA Vanco $_{5 \%}+\mathrm{Rif}_{0.5 \%}$-CPS/PLCA

Vanco Va $_{5 \%}+$ Rif $_{0.05 \%}$-CPS

Fig. 10. After 3 weeks in vivo, the retrieved scaffolds were vortexed in $1 \mathrm{~mL}$ PBS and filter paper discs were allowed to adsorb the eluate overnight before placing the discs onto Xen36 inoculated Luria agar plates (a). Only eluate from the $\mathrm{Vanco}_{5 \%}+\mathrm{Rif}_{0.5 \%}$-CPS/PLGA samples produced a bacterial inhibition zone around the respective disc. Note the lack of positive bioluminescent radiance surrounding the $\mathrm{Vanco}_{5 \%}+\mathrm{Rif}_{0.5 \%}$-CPS/PLGA sample. (b) Only the $\mathrm{Vanco}_{5 \%}+\mathrm{Rif}_{0.5 \%}-\mathrm{CPS} / \mathrm{PLGA}$ group had significantly reduced radiance values around the disc compared to the placebo PMMA sample (PBO-PMMA). *denotes $p<0.05 v s$. PBO-PMMA by Dunnett's test after ANOVA. $n=4 /$ group in duplicates.

sustained release CPS/PLGA treatment compared with the vancomycin-laden PMMA $(2.1 \pm 2.3 v s .5 .2 \pm 0.8 \mathrm{log}$ $(1+\mathrm{CFU} / \mathrm{g})$, respectively; $p=0.002$; Fig. 9a). The bones were bacteria culture negative in $50 \%(4 / 8)$ of the mice that received $\mathrm{Vanco}_{5 \%}+\mathrm{Rif}_{05 \%}-\mathrm{CPS} / \mathrm{PLGA}, 25 \%(2 / 8)$ that received Vanco $_{5 \%}+$ Rif $_{0.5 \%}$-CPS, $12.5 \%(1 / 8)$ that received $\mathrm{Vanco}_{5 \%}+\mathrm{Rif}_{0.05}$-CPS, and $0 \%(0 / 15)$ that received Vanco $_{5 \%}$-PMMA (Fig. 9a). All of the rifampinladen CPS treatments significantly reduced the bacterial burden in the soft tissue relative to Vanco $_{5 \%}$-PMMA as well $(1.0 \pm 1.3$ vs. $3.8 \pm 1.4 \log (1+\mathrm{CFU} / \mathrm{g}) ; p<0.003)$, which were $63 \%$ culture negative within each CPS group (Fig $9 b)$. The PMMA spacers were susceptible to colonisation by the bacteria, with $60 \%(9 / 15)$ of the implants culture positive at the end of the study (Fig. 9c). In contrast, the Vanco $_{5 \%}+\mathrm{Rif}_{0.5 \%}-\mathrm{CPS}$ and $\mathrm{Vanco}_{5 \%}+\mathrm{Rif}_{0.5 \%}-\mathrm{CPS} / \mathrm{PLGA}$ formulations were $100 \%$ culture negative (Fig. 9c). In fact, the retrieved Vanco $_{5 \%}+$ Rif $_{0.5 \%}$-CPS/PLGA still released active antibiotics at the end of the study (Fig. 10), which suggests that these CPS would deliver antibiotics for more than 3 weeks in vivo. Bacteria were not eradicated from the fixation plates and screws in any of the mice, but the bacterial load was significantly reduced by local rifampin therapy compared to Vanco $_{5 \%}-\mathrm{PMMA}(4.7 \pm 0.9$ vs. $5.9 \pm 0.7 \log (1+\mathrm{CFU} / \mathrm{g}) ; p=0.03$; Fig. $9 \mathrm{~d})$.

The osteolytic bone resorption was significantly reduced by the higher rifampin dose $(20.0 \pm 7.7 \%$ resorption; $p=0.045$ ) and the sustained release CPS 
a

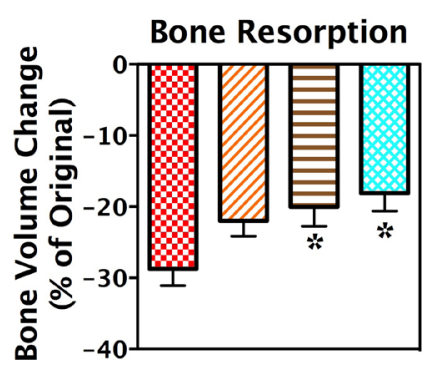

b

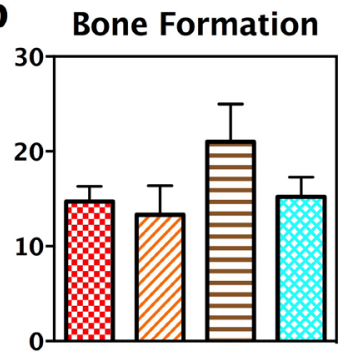

C Net Bone Change

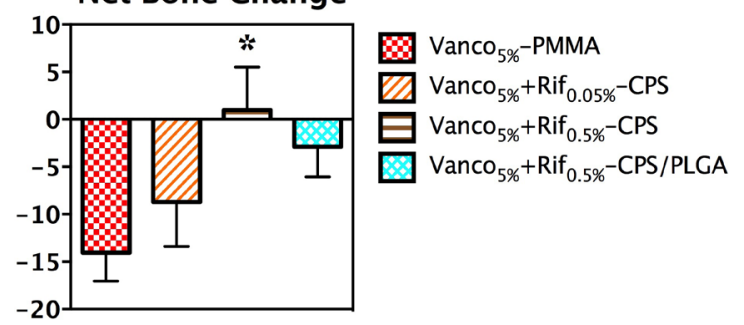

Fig. 11. Infection-induced bone loss is significantly reduced in mice that were treated with the higher rifampin dose or extended release formulations. Volumetric bone changes were measured by overlaying micro-CT scans that were collected one day after the revision surgery (day 8) and on the last day of the study (day 28). (a) The higher rifampin dose and sustained release groups significantly reduced the volumetric bone resorption compared with Vanco ${ }_{5 \%}-\mathrm{PMMA}$. (b) No significant differences in bone formation were observed. (c) The net bone change was significantly more positive in the $\mathrm{Vanco}_{5 \%}+\mathrm{Rif}_{0.5 \%}$-CPS group. *denotes multiplicity-adjusted $p<0.05 v s$. Vanco ${ }_{5 \%}-\mathrm{PMMA}^{*}$ by Dunnett's test after ANOVA.

formulations $(18.1 \pm 7.1 \%$ resorption; $p=0.012)$ compared to Vanco $_{5 \%}$-PMMA $(28.8 \pm 9.3 \%$ resorption; Fig. $11 \mathrm{~b})$. Similar levels of reactive bone formation were observed across all of the treatment groups (Fig. 11c), and the higher rifampin dose achieved a significantly more positive net bone change compared to $\operatorname{Vanco}_{5 \%}-\mathrm{PMMA}(+1.0 \pm 12.8 \%$ vs. $-14.0 \pm 12.0 \%$ net change; $p=0.017$; Fig. $11 \mathrm{~d})$.

\section{Discussion}

Treatment of implant-associated bone infections remains a significant clinical challenge despite the aggressive surgical and antibiotic treatments that are currently used. The general advantages of local antibiotic delivery are well established for osteomyelitis (Gogia et al., 2009), but the potential efficacy of local rifampin therapy against an implant-associated bone infection with biofilm involvement had not previously been studied. In vitro studies (Mihailescu et al., 2014; Parra-Ruiz et al., 2010; Saginur et al., 2006; Tang et al., 2013) have demonstrated evidence for rifampin's efficacy against $S$. aureus in biofilms. Studies in experimental animal models (Garrigos et al., 2010; John et al., 2009; Niska et al., 2013; Saleh-Mghir et al., 2011; Vergidis et al., 2011) and clinical studies (Drancourt et al., 1997; Widmer et al., 1992; Zimmerli et al., 1998) have further corroborated the benefits of rifampin, administered systemically, in cases of implant-associated staphylococcal infections with likely biofilm involvement. Therefore, this study examined the concomitant local delivery of vancomycin and rifampin from 3D printed CPS for treatment of a Staphylococcal bone infection in the presence of an orthopaedic implant contaminated with biofilm.

Considering the potential toxicity of rifampin to local osteogenic cells (Duewelhenke et al., 2007; Rathbone et $a l ., 2011)$ and the very low MIC for planktonic $S$. aureus $(0.08 \mu \mathrm{g} / \mathrm{mL}$ (Mihailescu et al., 2014)), we first evaluated a low dose of rifampin $(0.05 \mathrm{wt} \%$ or $3.5 \mu \mathrm{g}$ per murine scaffold). Even with this low rifampin dose, significant reductions in the bacterial load and osteolysis were observed compared with vancomycin-laden PMMA. Yet, the higher rifampin dose and sustained release formulations tended to further enhance the efficacy of the rifampinladen CPS. Although the differences in mean CFU were not statistically significant, the Vanco $_{5 \%}+$ Rif $_{0.5} \%$ CPS and $\mathrm{Vanco}_{5 \%}+\mathrm{Rif}_{0.5 \%}$-CPS/PLGA resulted in higher rates of culture negative bones compared with the lower dose Vanco $_{5 \%}+\mathrm{Rif}_{0.05 \%}$-CPS. In addition, the retrieved Vanco $_{5 \%}+\mathrm{Rif}_{0.5} \%-\mathrm{CPS} / \mathrm{PLGA}$ scaffolds were still bactericidal at the end of the 3 week treatment study. In contrast, the PMMA spacers often became inert after the initial elution of antibiotics from the surface and were susceptible to colonisation by the remaining bacteria. This problem was observed in $60 \%(9 / 15)$ of the mice that received PMMA in this study and has also been documented clinically (Mariconda et al., 2013; Schmolders et al., 2014).

Previous in vitro studies have found that rifampin is more cytotoxic to primary human osteoblasts than many other antibiotics (Duewelhenke et al., 2007; Rathbone et al., 2011). In this septic bone defect study, reactive bone formation was not significantly reduced by any of the rifampin treatments compared to vancomycin-laden PMMA. These data suggest that the local rifampin delivery, regardless of dose or elution time in this study, did not significantly impair the activity of local osteogenic cells.

The significant reduction in BLI signal from day 7 post-op to day 10 in the groups that received local rifampin, and the lack thereof in every vancomycin monotherapy group (including Vanco $_{5 \%}$-CPS), suggests that rifampin may be important for attenuating the bacterial metabolic load shortly after the initiation of treatment. The use of BLI in this infection model is limited to quantification of metabolically active bacteria, which may not include the more quiescent viable bacteria within the biofilm. Therefore, these BLI data can only evidence that rifampin better controls the planktonic bacteria following revision. A limitation to this comparison is that systemic antibiotic therapy would have been initiated prior to the revision 
surgery in a clinical situation, which may have resulted in a lower BLI signal in the vancomycin monotherapy cases since new planktonic bacteria that could be released during debridement likely would have been controlled by pre-existing systemic antibiotic. Nonetheless, the observed benefits of local rifampin and vancomycin co-therapy are consistent with many in vitro (Mihailescu et al., 2014; Parra-Ruiz et al., 2010; Saginur et al., 2006; Tang et al., 2013) and in vivo (Garrigos et al., 2010; John et al., 2009; Niska et al., 2013; Saleh-Mghir et al., 2011; Vergidis et al., 2011) studies, which have found that combinational therapies that include rifampin exceed the efficacy of each monotherapy.

Although the local rifampin delivery significantly reduced the mean bacterial load and produced higher rates of culture negative tissues, culture positive mice still existed in each treatment group and $100 \%$ of the fixation plates or screws were culture positive at the end of the study. As in every infection treatment study, the culture negative sample rate is an important factor considering that residual viable bacteria will pose a risk for re-infection after cessation of treatment. Yet, complete eradication is extremely challenging in implant-related $S$. aureus infections. Many other pre-clinical models of implant-associated $S$. aureus infections were also refractory to conventional antibiotic treatments that employed systemic rifampin combined with vancomycin, daptomycin, linezolid, or levofloxacin, or the respective monotherapies (Garrigos et al., 2010; John et al., 2009; Niska et al., 2013; Saleh-Mghir et al., 2011; Vergidis et al., 2011). Only one study observed a $100 \%$ clearance, which used daptomycin plus rifampin against a knee periprosthetic infection model in rabbits (Saleh-Mghir et al., 2011). Niska et al. systemically administered rifampin, in combination with vancomycin, to a mouse model with a femoral intramedullary pin, with a Xen36 infection in the knee joint and found $100 \%$ clearance of bacteria from the pins and $67 \%$ clearance from the peri-implant tissue (Niska et al., 2013). However, in contrast to the current study, Niska et al. measured approximately 500 CFU per pin and only a $67 \%$ culture positive rate on the pins in the untreated group, which is extremely low compared to the $6 \times 10^{6} \mathrm{CFU}$ and $100 \%$ colonisation in the untreated group of the trauma infection model utilised herein (Inzana et al., 2015). The fundamental differences between these knee joint peri-prosthetic models and the current trauma model, as well as the differences in CFU counts in untreated animals, makes direct comparison with the current results very challenging. Evaluating systemic rifampin plus vancomycin in the current model may have provided some additional insights, but the current study was focused on local delivery strategies. Since the goal is to achieve a high concentration of antibiotics in the infected tissues for a sufficient time, the origin of the antibiotic (systemic injection $v s$. local reservoir) may be less important than the resulting bioavailability at the infection site. Nonetheless, these current and previous results highlight the extreme challenge of treating biofilms and caution against implant retention in the presence of a biofilm-forming species.

This challenging infection model may have been refractory to the current treatments if the vancomycinrifampin combination was not sufficiently effective against the bacteria in biofilm or the concentrations achieved by the antibiotics were still sub-lethal. Indeed, the MIC of rifampin against $S$. aureus in biofilm is orders of magnitude greater than planktonic $S$. aureus $(164 \mu \mathrm{g} / \mathrm{mL}$ vs. $0.08 \mu \mathrm{g} /$ $\mathrm{mL}$ (Mihailescu et al., 2014)). Yet, it is important to note that the antibiotic concentrations measured in vitro (Fig. 7) are likely to be lower than the in vivo concentrations based on the local fluid volumes in each scenario. Vancomycin was chosen as the co-therapy for this study based on its common clinical use against MRSA strains (Deresinski, 2009). Although Xen36 is methicillin-sensitive, the vancomycin MIC for Xen36 is no more than $0.5 \mu \mathrm{g} /$ $\mathrm{mL}$ (Niska et al., 2013), compared with $1-2 \mu \mathrm{g} / \mathrm{mL}$ in a variety of MRSA strains (Crandon et al., 2010). Clinically, however, Trampuz and Zimmerli recommend systemically administered rifampin in combination with (flu)cloxacillin for methicillin-sensitive Staphylococci or rifampin plus vancomycin for MRSA, followed by rifampin plus ciprofloxacin or levofloxacin after the first two weeks (Trampuz and Zimmerli, 2005; Trampuz and Zimmerli, 2006). Other antibiotic combinations with rifampin may have been more effective against the bacteria in biofilm than vancomycin (Garrigos et al., 2010; John et al., 2009; Mihailescu et al., 2014; Parra-Ruiz et al., 2010; Tang et al., 2013), but the most efficacious antibiotic combination is inconclusive when considering all of the previous in vitro and in vivo studies together. The similar results from treatment with Vanco $_{5 \%}$-CPS and Vanco ${ }_{5 \%}$-PMMA suggest that the delivery material was less important than the choice of antibiotic in this study. An important future study would be to evaluate these vancomycin- and rifampin-laden CPS in a similar animal model, but with complete exchange of the fixation hardware during the revision surgery, to determine if recolonisation of the new plate and screws can be prevented.

While this study demonstrates the specific efficacy of a rifampin- and vancomycin-laden CPS in a murine infection model, the additive manufacturing techniques used herein are highly versatile and easily amenable to the inclusion of alternative antibiotics or extension to other regenerative medicine and tissue engineering applications. As demonstrated in this study, the 3D printing process readily provides adaptive modularity through alternative bioactive 'inks' that can be infused using the inkjets (e.g. biofilm dispersal agents to augment the antibiotic efficacy), through variations in the powder phase composition, and through post-printing modifications to the scaffold. This versatility is extended by the expanse of possible geometries as well as the selective spatial distribution of bioactive molecules within each layer, which may provide additional control over the release kinetics (Klammert et al., 2010) or site-specific scaffold bioactivity (Gbureck et al., 2007a). Following successful infection management, the osteoconductive 3D printed CPS may serve as a scaffold for regeneration of the bone defect without a second revision surgery, as is necessary with the use of PMMA. Finally, 3D printing enables easily customisable solutions for medical image-guided, patient-specific therapy, which will greatly facilitate future clinical translation, especially for craniomaxillofacial applications in which the aesthetic aspects of the reconstruction are important. 


\section{Conclusions}

In this study, we have demonstrated that the concomitant local delivery of rifampin and vancomycin from $3 \mathrm{D}$ printed CPS significantly improves the outcomes of an implantrelated bone infection in a mouse model compared to the current clinical practice of using PMMA spacers, which cannot carry rifampin. The higher rifampin dose and postprinting PLGA coating further enhanced the efficacy of these materials through prolonged local antibiotic delivery to successfully manage the infection and potentially eradicate the pathogen from the bone in $50 \%$ of the mice. Following successful infection management, the osteoconductive $3 \mathrm{D}$ printed CPS may serve as a scaffold for regeneration of the bone defect without a second revision surgery, as is necessary with the use of PMMA. Yet, based on these current results and those of many other studies discussed herein, it seems that the only way to completely cure an infection is to remove all of the bacteria and biofilm (implant exchange and aggressive debridement), or induce the bacteria to return to a planktonic state where they are more susceptible to antimicrobials (the goal of experimental biofilm dispersal agents (Kaplan, 2010)). We are not convinced that a particular antibiotic combination or dosage alone will assuredly kill $100 \%$ of the bacteria, although the clinical symptoms would likely be suppressed.

\section{Acknowledgements}

The Authors would like to thank Romano Matthys (RISystem) for custom designing the titanium-coated PEEK fixation plates, Michael Thullen (University of Rochester) for his assistance with micro-CT, Karen Bentley (University of Rochester) for her expertise in scanning electron microscopy, as well as Luiz Meirelles, Barbra LaRue, and Sarah Mack (University of Rochester) for their assistance with histology. This study was supported by the AOTrauma Research- Clinical Priority Program on Bone Infection and NIAMS/NIH grant P30AR061307. Jason Inzana was supported by an NSF graduate research fellowship (NSF Award DGE-1419118). The content is solely the responsibility of the authors and does not necessarily represent the official views of the National Science Foundation, National Institutes of Health, or AOTrauma.

\section{References}

Ahola N, Veiranto M, Mannisto N, Karp M, Rich J, Efimov A, Seppala J, Kellomaki M (2012) Processing and sustained in vitro release of rifampicin containing composites to enhance the treatment of osteomyelitis. Biomatter 2: 213-225.

Beeching NJ, Thomas MG, Roberts S, Lang SD (1986) Comparative in-vitro activity of antibiotics incorporated in acrylic bone cement. J Antimicrob Chemother 17: 173-184.

Bonev B, Hooper J, Parisot J (2008) Principles of assessing bacterial susceptibility to antibiotics using the agar diffusion method. J Antimicrob Chemother 61: 12951301.

Bose S, Tarafder S (2011) Calcium phosphate ceramic systems in growth factor and drug delivery for bone tissue engineering: A review. Acta Biomater 8: 1401-1421.

Crandon JL, Kuti JL, Nicolau DP (2010) Comparative efficacies of human simulated exposures of telavancin and vancomycin against methicillin-resistant Staphylococcus aureus with a range of vancomycin MICs in a murine pneumonia model. Antimicrobial agents and chemotherapy 54: 5115-5119.

Darouiche RO (2004) Treatment of infections associated with surgical implants. N Engl J Med 350: 1422-1429.

Darouiche RO, Mansouri MD, Zakarevicz D, Alsharif A, Landon GC (2007) In vivo efficacy of antimicrobialcoated devices. J Bone Joint Surg Am 89: 792-797.

Deresinski S (2009) Vancomycin in combination with other antibiotics for the treatment of serious methicillinresistant Staphylococcus aureus infections. Clin Infect Dis 49: 1072-1079.

Drancourt M, Stein A, Argenson JN, Roiron R, Groulier P, Raoult D (1997) Oral treatment of Staphylococcus spp. infected orthopaedic implants with fusidic acid or ofloxacin in combination with rifampicin. J Antimicrob Chemother 39: $235-240$.

Duewelhenke N, Krut O, Eysel P (2007) Influence on mitochondria and cytotoxicity of different antibiotics administered in high concentrations on primary human osteoblasts and cell lines. Antimicrob Agents Chemother 51: 54-63.

Garrigos C, Murillo O, Euba G, Verdaguer R, Tubau F, Cabellos C, Cabo J, Ariza J (2010) Efficacy of usual and high doses of daptomycin in combination with rifampin versus alternative therapies in experimental foreign-body infection by methicillin-resistant Staphylococcus aureus. Antimicrob Agents Chemother 54: 5251-5256.

Gbureck U, Hölzel T, Doillon CJ, Müller FA, Barralet JE (2007a) Direct printing of bioceramic implants with spatially localized angiogenic factors. Advanced materials 19: $795-800$.

Gbureck U, Hölzel T, Klammert U, Würzler K, Müller FA, Barralet JE (2007b) Resorbable dicalcium phosphate bone substitutes prepared by 3D powder printing. Advanced Functional Materials 17: 3940-3945.

Ginebra MP, Traykova T, Planell JA (2006) Calcium phosphate cements as bone drug delivery systems: a review. J Control Release. J Control Release 113: 102-110.

Gogia JS, Meehan JP, Di Cesare PE, Jamali AA (2009) Local antibiotic therapy in osteomyelitis. Semin Plast Surg 23: 100-107.

Gu Y, Chen X, Lee JH, Monteiro DA, Wang H, Lee WY (2012) Inkjet printed antibiotic- and calcium-eluting bioresorbable nanocomposite micropatterns for orthopedic implants. Acta Biomater 8: 424-431.

Inzana JA, Olvera D, Fuller SM, Kelly JP, Graeve OA, Schwarz EM, Kates SL, Awad HA (2014) 3D printing of composite calcium phosphate and collagen scaffolds for bone regeneration. Biomaterials 35: 4026-4034.

Inzana JA, Schwarz EM, Kates SL, Awad HA (2015) A novel murine model of established Staphylococcal bone 
infection in the presence of a fracture fixation plate to study therapies utilizing antibiotic-laden spacers after revision surgery. Bone 72: 128-136.

Jaeblon T (2010) Polymethylmethacrylate: properties and contemporary uses in orthopaedics. J Am Acad Orthop Surg 18: 297-305.

John AK, Baldoni D, Haschke M, Rentsch K, Schaerli P, Zimmerli W, Trampuz A (2009) Efficacy of daptomycin in implant-associated infection due to methicillin-resistant Staphylococcus aureus: importance of combination with rifampin. Antimicrob Agents Chemother 53: 2719-2724.

Kaplan JB (2010) Biofilm dispersal: mechanisms, clinical implications, and potential therapeutic uses. J Dent Res 89: 205-218.

Keren I, Kaldalu N, Spoering A, Wang Y, Lewis K (2004) Persister cells and tolerance to antimicrobials. FEMS Microbiol Lett 230: 13-18.

Klammert U, Gbureck U, Vorndran E, Rodiger J, Meyer-Marcotty P, Kubler AC (2010) 3D powder printed calcium phosphate implants for reconstruction of cranial and maxillofacial defects. J Craniomaxillofac Surg 38: 565-570.

Konig DP, Schierholz JM, Munnich U, Rutt J (2001) Treatment of staphylococcal implant infection with rifampicin-ciprofloxacin in stable implants. Arch Orthop Trauma Surg 121: 297-299.

Mariconda M, Ascione T, Balato G, Rotondo R, Smeraglia F, Costa GG, Conte M (2013) Sonication of antibiotic-loaded cement spacers in a two-stage revision protocol for infected joint arthroplasty. BMC Musculoskelet Disord 14: 193.

Mihailescu R, Furustrand Tafin U, Corvec S, Oliva A, Betrisey B, Borens O, Trampuz A (2014) High activity of fosfomycin and rifampin against methicillinresistant Staphylococcus aureus biofilm in vitro and in an experimental foreign-body infection model. Antimicrobial agents and chemotherapy 58: 2547-2553.

Molina-Manso D, Manzano M, Doadrio JC, Del Prado G, Ortiz-Perez A, Vallet-Regi M, Gomez-Barrena E, Esteban J (2012) Usefulness of SBA-15 mesoporous ceramics as a delivery system for vancomycin, rifampicin and linezolid: a preliminary report. Int J Antimicrob Agents 40: $252-256$.

Niska JA, Shahbazian JH, Ramos RI, Francis KP, Bernthal NM, Miller LS (2013) Vancomycin-rifampin combination therapy has enhanced efficacy against an experimental Staphylococcus aureus prosthetic joint infection. Antimicrob Agents Chemother 57: 5080-5086.

O'hara P, Hickey AJ (2000) Respirable PLGA microspheres containing rifampicin for the treatment of tuberculosis: manufacture and characterization. Pharm Res 17: 955-961.

Parra-Ruiz J, Vidaillac C, Rose WE, Rybak MJ (2010) Activities of high-dose daptomycin, vancomycin, and moxifloxacin alone or in combination with clarithromycin or rifampin in a novel in vitro model of Staphylococcus aureus biofilm. Antimicrobial agents and chemotherapy 54: 4329-4334.

Pribaz JR, Bernthal NM, Billi F, Cho JS, Ramos RI, Guo Y, Cheung AL, Francis KP, Miller LS (2012) Mouse model of chronic post-arthroplasty infection: noninvasive in vivo bioluminescence imaging to monitor bacterial burden for long-term study. J Orthop Res 30: 335-340.

Rani SA, Pitts B, Beyenal H, Veluchamy RA, Lewandowski Z, Davison WM, Buckingham-Meyer K, Stewart PS (2007) Spatial patterns of DNA replication, protein synthesis, and oxygen concentration within bacterial biofilms reveal diverse physiological states. J Bacteriol 189: 4223-4233.

Rathbone CR, Cross JD, Brown KV, Murray CK, Wenke JC (2011) Effect of various concentrations of antibiotics on osteogenic cell viability and activity. J Orthop Res 29: 1070-1074.

Saginur R, Stdenis M, Ferris W, Aaron SD, Chan F, Lee C, Ramotar K (2006) Multiple combination bactericidal testing of staphylococcal biofilms from implant-associated infections. Antimicrob Agents Chemother 50: 55-61.

Saleh-Mghir A, Muller-Serieys C, Dinh A, Massias L, Cremieux AC (2011) Adjunctive rifampin is crucial to optimizing daptomycin efficacy against rabbit prosthetic joint infection due to methicillin-resistant Staphylococcus aureus. Antimicrob Agents Chemother 55: 4589-4593.

Schmolders J, Hischebeth GT, Friedrich MJ, Randau TM, Wimmer MD, Kohlhof H, Molitor E, Gravius S (2014) Evidence of MRSE on a gentamicin and vancomycin impregnated polymethyl-methacrylate (PMMA) bone cement spacer after two-stage exchange arthroplasty due to periprosthetic joint infection of the knee. BMC Infect Dis 14: 144.

Tang HJ, Chen CC, Cheng KC, Wu KY, Lin YC, Zhang CC, Weng TC, Yu WL, Chiu YH, Toh HS, Chiang SR, Su BA, Ko WC, Chuang YC (2013) In vitro efficacies and resistance profiles of rifampin-based combination regimens for biofilm-embedded methicillin-resistant Staphylococcus aureus. Antimicrob Agents Chemother 57: 5717-5720.

Trampuz A, Widmer AF (2006) Infections associated with orthopedic implants. Curr Opin Infect Dis 19: 349356.

Trampuz A, Zimmerli W (2005) Prosthetic joint infections: update in diagnosis and treatment. Swiss Med Wkly 135: 243-251.

Trampuz A, Zimmerli W (2006) Diagnosis and treatment of infections associated with fracture-fixation devices. Injury 37 Suppl 2: S59-66.

Trebse R, Pisot V, Trampuz A (2005) Treatment of infected retained implants. J Bone Joint Surg Br Volume 87: 249-256.

Valour F, Karsenty J, Bouaziz A, Ader F, Tod M, Lustig S, Laurent F, Ecochard R, Chidiac C, Ferry T, Lyon BJISG (2014) Antimicrobial-related severe adverse events during treatment of bone and joint infection due to methicillinsusceptible Staphylococcus aureus. Antimicrob Agents Chemother 58: 746-755.

Vergidis P, Rouse MS, Euba G, Karau MJ, Schmidt SM, Mandrekar JN, Steckelberg JM, Patel R (2011) Treatment with linezolid or vancomycin in combination with rifampin is effective in an animal model of methicillin-resistant Staphylococcus aureus foreign body osteomyelitis. Antimicrob Agents Chemother 55: 1182-1186.

Vorndran E, Klammert U, Ewald A, Barralet JE, Gbureck U (2010) Simultaneous Immobilization of Bioactives During 3D Powder Printing of Bioceramic 
Drug-Release Matrices. Advanced Functional Materials 20: 1585-1591.

Waeiss RA, Negrini TC, Arthur RA, Bottino MC (2014) Antimicrobial effects of drug-containing electrospun matrices on osteomyelitis-associated pathogens. J Oral Maxillofac Surg 72: 1310-1319.

Widmer AF, Gaechter A, Ochsner PE, Zimmerli W (1992) Antimicrobial treatment of orthopedic implantrelated infections with rifampin combinations. Clin Infect Dis 14: 1251-1253. Yun HC, Branstetter JG, Murray CK (2008) Osteomyelitis in military personnel wounded in Iraq and Afghanistan J Trauma 64: S163-168; discussion S168.

Zimmerli W, Trampuz A, Ochsner PE (2004) Prosthetic-joint infections N Engl J Med 351: 1645-1654.

Zimmerli W, Widmer AF, Blatter M, Frei R, Ochsner PE (1998) Role of rifampin for treatment of orthopedic implant-related staphylococcal infections: a randomized controlled trial. Foreign-Body Infection (FBI) Study Group JAMA 279: 1537-1541.

\section{Discussion with Reviewers}

Joseph Wenke: Rifampin has gained a lot of interest lately due to its effectiveness against biofilms. What are some of the experiments that must be performed to determine if and how to use it in a locally-applied manner in open fractures? Authors: Rifampin likely should not be used as a prophylactic agent considering that bacteria develop resistance to this antibiotic more rapidly than most others. The results of the current study provide some of the first evidence for the therapeutic efficacy of locally administered rifampin for an established bone infection in a rigorously evaluated preclinical model. Surely, further experimentation would be beneficial to improve our understanding of whether rifampin can completely eradicate an infection when combined with vancomycin or another effective antibiotic (e.g. linezolid, daptomycin, etc.). The required concentrations and release kinetics, which were not $100 \%$ effective herein, would need to be determined. The systemic serum concentrations would also need to be monitored in larger animals or humans to ensure that overdosing does not occur, although this would be unlikely from a local delivery strategy. As mentioned in this article, local rifampin delivery will not be possible with PMMA beads or spacers unless the PMMA is augmented to tolerate this antibiotic, or the antibiotic is isolated in microspheres, or some similar strategy is used. 3D printed calcium phosphate spacers were studied herein, but such a specific material is not required to further study the potential of locally administered rifampin. It is important to recognise that local rifampin therapy is not hypothesised to be a substitute for implant-exchange and that implant exchange during revision will always be a highly effective way to minimise the risk of recurrent infection due to viable biofilm. Therefore, this murine model is an extremely challenging case and represents a worse case scenario due to the retention of the biofilm ridden fixation hardware. In line with this point, an important future study would also examine the efficacy of locally administered rifampin in a pre-clinical model of revision with complete implant exchange. However, this may not be possible in the mouse model utilised herein, based on the minimal bone stock for new screw anchorage in these very small bones. To summarise, some important future questions to address would be:

- If systemic rifampin therapy can completely eradicate the infection, what are the tissue concentrations over time around the infection site that could enable this success?

- Subsequently, can a local delivery device be designed to achieve the same concentrations?

- Can local rifampin therapy, when combined with another antibiotic, prevent recolonisation and new biofilm formation after a revision surgery with complete implant exchange?

- Is there an optimal antibiotic choice to complement rifampin and should this be delivered systemically or locally?

David Grainger: Bacterial colony reduction using your approach is much more dramatic for soft tissue than it is for hardware or bone, and better correlated to mitigating infection in your model. Do you think that soft tissue elimination of bacteria is a better predictor of infection severity and risk for repeated infection over bone and hardware colonisation assessments? Should more attention be better paid to soft tissue health/morbidity/antibiotic load surrounding implants in addressing infection risks and treatment efficacies?

Authors: Maintaining healthy soft tissue is critical for infection management and is an important focus area. Healthy vascularised tissue is important to continue supplying the infection site with systemic antibiotics and immune cells as well as for successful closure of the wound. Likewise, we agree that it is very likely that the clinical severity of these infections may be more directly related to the soft tissue infection. A severe soft tissue infection likely increases the risk of unsuccessful clinical management due, in part, to higher levels of inflammation and a lack of wound closure; but it is more likely that the risk of recurrent infection is associated with the more insidious bacterial colonisation of the bone or within biofilm that does not elicit a strong host response, yet could provide a reservoir for re-establishing a clinically apparent infection.

Fintan T Moriarty: The primary reason to use rifampicin in treatment of implant-associated infection is due to its proven anti-biofilm activity. The results presented in this study show that locally delivered rifampicin improves eradication of bacteria from the tissue, but does not eradicate the biofilm on the implant. Therefore, do you think the rifampicin does not function to its maximal potential effect when delivered locally, and could it be that simply the combination of two antibiotics is better than one antibiotic (and rifampicin is not needed in this situation at all)?

Authors: Indeed, the strong evidence for the effects of rifampin against bacteria in biofilm primarily motivate its use in implant-associated infections. However, suggesting 
that rifampin, or any other drug, cannot function to its maximal effect when delivered locally is an overgeneralisation. The bioavailability of the antibiotic at the infection site is the key factor. Perhaps the local delivery dosages or release profiles that were used in this study were non-optimal to achieve effective tissue concentrations for an appropriate period. The dosages and release kinetics of a local delivery strategy can be tuned through the properties and drug loading of the delivery material, just as systemic administration can be tuned by providing different doses at different times through different routes (e.g. intravenous vs. oral).

It is also possible that the anti-biofilm activity of rifampin is not entirely robust. In this manuscript, we discuss five studies other than the current study that were, at least in part, refractory to combinational antibiotic treatments that included rifampin, delivered systemically. These studies also showed that combining antibiotics such as vancomycin, daptomycin, linezolid, or levofloxacin with rifampin consistently performed better than the respective monotherapy. It is possible that if vancomycin was combined with another antibiotic in this study other than rifampin, this alternative combination may have yielded better results than vancomycin monotherapy as well. Selection of antibiotics and combinations can be very challenging. Given the wide variety of preclinical animal models and variability in clinical studies, conclusive quantitative comparisons of different antibiotic combinations are difficult. Interested readers are referred to a much more comprehensive review on antibiotic selection for osteomyelitis (Fraimow et al., 2009), considering that this topic quickly evolves beyond the scope of this current study.

\section{Additional Reference}

Fraimow HS (2009) Systemic antimicrobial therapy in osteomyelitis. Semin Plast Surg 23: 90-99.

Editor's Note: Scientific Editor in charge of the paper: Geoff Richards 
